\title{
Annealed deviations of random walk in random scenery
}

\author{
Nina Gantert ${ }^{a}$, Wolfgang König ${ }^{\mathrm{b}, *}$, Zhan Shi ${ }^{\mathrm{c}}$ \\ a Fachbereich Mathematik und Informatik der Universität Münster, Einsteinstraße 62, 48149 Münster, Germany \\ $\mathrm{b}^{\mathrm{b}}$ Mathematisches Institut, Universität Leipzig, Augustusplatz 10/11, 04109 Leipzig, Germany \\ ${ }^{\mathrm{c}}$ Laboratoire de Probabilités et Modèles Aléatoires, Université Paris VI, 4, place Jussieu, 75252 Paris Cedex 05, France
}

Received 4 April 2005; received in revised form 21 November 2005; accepted 20 December 2005

Available online 7 July 2006

\begin{abstract}
Let $\left(Z_{n}\right)_{n \in \mathbb{N}}$ be a $d$-dimensional random walk in random scenery, i.e., $Z_{n}=\sum_{k=0}^{n-1} Y\left(S_{k}\right)$ with $\left(S_{k}\right)_{k \in \mathbb{N}_{0}}$ a random walk in $\mathbb{Z}^{d}$ and $(Y(z))_{z \in \mathbb{Z}^{d}}$ an i.i.d. scenery, independent of the walk. The walker's steps have mean zero and some finite exponential moments. We identify the speed and the rate of the logarithmic decay of $\mathbb{P}\left(\frac{1}{n} Z_{n}>b_{n}\right)$ for various choices of sequences $\left(b_{n}\right)_{n}$ in $[1, \infty)$. Depending on $\left(b_{n}\right)_{n}$ and the upper tails of the scenery, we identify different regimes for the speed of decay and different variational formulas for the rate functions. In contrast to recent work [A. Asselah, F. Castell, Large deviations for Brownian motion in a random scenery, Probab. Theory Related Fields 126 (2003) 497-527] by A. Asselah and F. Castell, we consider sceneries unbounded to infinity. It turns out that there are interesting connections to large deviation properties of self-intersections of the walk, which have been studied recently by X. Chen [X. Chen, Exponential asymptotics and law of the iterated logarithm for intersection local times of random walks, Ann. Probab. 32 (4) 2004].
\end{abstract}

(c) 2006 Elsevier Masson SAS. All rights reserved.

\section{Résumé}

Soit $\left(Z_{n}\right)_{n \in \mathbb{N}}$ une marche aléatoire en paysage aléatoire sur $\mathbb{Z}^{d}$; il s'agit du processus défini par $Z_{n}=\sum_{k=0}^{n-1} Y\left(S_{k}\right)$, où $\left(S_{k}\right)_{k \in \mathbb{N}_{0}}$ est une marche aléatoire à valeurs dans $\mathbb{Z}^{d}$, et le paysage aléatoire $(Y(z))_{z \in \mathbb{Z}^{d}}$ est une famille de variables aléatoires i.i.d. indépendante de la marche. On suppose que $S_{1}$ est centrée et admet certains moments exponentiels finis. Nous identifions la vitesse et la fonction de taux de $\mathbb{P}\left(\frac{1}{n} Z_{n}>b_{n}\right)$, pour diverses suites $\left(b_{n}\right)_{n}$ à valeurs dans $\left[1, \infty\left[\right.\right.$. Selon le comportement de $\left(b_{n}\right)_{n}$ et de la queue de distribution du paysage aléatoire, nous découvrons différents régimes ainsi que différentes formules variationnelles pour les fonctions de taux. Contrairement au travail récent de A. Asselah et F. Castell [A. Asselah, F. Castell, Large deviations for Brownian motion in a random scenery, Probab. Theory Related Fields 126 (2003) 497-527], nous étudions le cas où le paysage aléatoire n'est pas borné. Finalement, nous observons des liens intéressants avec certaines propriétés d'auto-intersection de la marche $\left(S_{k}\right)_{k \in \mathbb{N}_{0}}$, récemment étudiées par X. Chen [X. Chen, Exponential asymptotics and law of the iterated logarithm for intersection local times of random walks, Ann. Probab. 32 (4) 2004].

(c) 2006 Elsevier Masson SAS. All rights reserved.

MSC: 60K37; 60F10; 60J55

Keywords: Random walk in random scenery; Local time; Large deviations; Variational formulas

\footnotetext{
* Corresponding author.

E-mail addresses: gantert@math.uni-muenster.de (N. Gantert), koenig@math.uni-leipzig.de (W. König), zhan@ proba.jussieu.fr (Z. Shi).
} 


\section{Introduction}

\subsection{Model and motivation}

Let $S=\left(S_{n}\right)_{n \in \mathbb{N}_{0}}$ be a random walk on $\mathbb{Z}^{d}$ starting at the origin. We denote by $\mathbb{P}$ the underlying probability measure and by $\mathbb{E}$ the corresponding expectation. We assume that $\mathbb{E}\left[S_{1}\right]=0$ and $\mathbb{E}\left[\left|S_{1}\right|^{2}\right]<\infty$. Defined on the same probability space, let $Y=(Y(z))_{z \in \mathbb{Z}^{d}}$ be an i.i.d. sequence of random variables, independent of the walk. We refer to $Y$ as the random scenery. Then the process $\left(Z_{n}\right)_{n \in \mathbb{N}}$ defined by

$$
Z_{n}=\sum_{k=0}^{n-1} Y\left(S_{k}\right), \quad n \in \mathbb{N},
$$

where $\mathbb{N}=\{1,2, \ldots\}$, is called a random walk in random scenery, sometimes also referred to as the Kesten-Spitzer random walk in random scenery, see [19]. An interpretation is as follows. If a random walker has to pay $Y(z)$ units at any time he/she visits the site $z$, then $Z_{n}$ is the total amount he/she pays by time $n-1$.

The random walk in random scenery has been introduced and analyzed for dimension $d \neq 2$ by $\mathrm{H}$. Kesten and F. Spitzer [19] and by E. Bolthausen [6] for $d=2$. The case $d=1$ was treated independently by A.N. Borodin [7,8]. Under the assumption that $Y(0)$ has expectation zero and variance $\sigma^{2} \in(0, \infty)$, their results imply that

$$
\frac{1}{n} Z_{n} \approx a_{n}^{(0)}= \begin{cases}n^{-1 / 4} & \text { if } d=1, \\ \left(\frac{n}{\log n}\right)^{-1 / 2} & \text { if } d=2, \\ n^{-1 / 2} & \text { if } d \geqslant 3 .\end{cases}
$$

More precisely, $\left(1 / n a_{n}^{(0)}\right) Z_{n}$ converges in distribution towards some non-degenerate random variable. The limit is Gaussian in $d \geqslant 2$ and a convex combination of Gaussians (but not Gaussian) in $d=1$. This can be roughly explained as follows. In terms of the so-called local times of the walk,

$$
\ell_{n}(z)=\sum_{k=0}^{n-1} \mathbb{1}_{\left\{S_{k}=z\right\}}, \quad n \in \mathbb{N}, z \in \mathbb{Z}^{d},
$$

the random walk in random scenery may be identified as

$$
Z_{n}=\sum_{z \in \mathbb{Z}^{d}} Y(z) \ell_{n}(z)
$$

The number of effective summands in (1.3) is equal to the range of the walk, i.e., the number of sites visited by time $n-1$. Hence, conditional on the random walk, $Z_{n}$ is, for dimension $d \geqslant 3$, a sum of $\mathcal{O}(n)$ independent copies of finite multiples of $Y(0)$, and hence it is plausible that $Z_{n} / n^{1 / 2}$ converges to a normal variable. The same assertion with logarithmic corrections is also plausible in $d=2$. However, in $d=1, Z_{n}$ is roughly a sum of $\mathcal{O}\left(n^{1 / 2}\right)$ copies of independent variables with variances of order $\mathcal{O}(n)$, and this suggests the normalization in (1.1) as well as a nonnormal limit.

In this paper, we analyze deviations $\left\{\frac{1}{n} Z_{n}>b_{n}\right\}$ for various choices of sequences $\left(b_{n}\right)_{n \in \mathbb{N}}$ in $[1, \infty)$. We determine the speed and the rate of the logarithmic asymptotics of the probability of this event as $n \rightarrow \infty$, and we explain the typical behavior of the random walk and the random scenery on this event.

This problem has been addressed in recent work [11,1] and [10] by F. Castell in partial collaboration with F. Pradeilles and A. Asselah for Brownian motion instead of random walk. While [11] and [10] treat the case of a continuous Gaussian scenery for $b_{n}=n^{1 / 2}$ and cst. $\leqslant b_{n} \ll n^{1 / 2}$, respectively, the case of an arbitrary bounded scenery (constant on the unit cubes) and $b_{n}=$ cst. is considered in [1]. See also [1] for further references on this topic and [2] and [16] for recent results on the random walk case.

The main novelty of the present paper is the study of arbitrary sceneries unbounded to $+\infty$ and general scale functions $b_{n} \geqslant$ cst. in the discrete setting. On the technical side, in particular the proof of the upper bound is rather demanding and requires new techniques. We solve this part of the problem by a careful analysis of high integer moments, a technique which has been recently established in the study of intersection properties of random motions.

A very rough, heuristic explanation of the interplay between the deviations of the random walk in random scenery and the tails of the scenery at infinity and the dimension $d$ is as follows. In order to realize the event $\left\{\frac{1}{n} Z_{n}>b_{n}\right\}$, it is 
clear that the scenery has to assume larger values on the range of the walk than usual. In order to keep the probabilistic cost for this low, the random walker has to keep its range small, i.e., it has to concentrate on less sites by time $n$ than usual. The optimal joint strategy of the scenery and the walk is determined by a balance between the respective costs. The optimal strategies in the cases considered in the present paper are homogeneous. More precisely, the scenery and the walk each approximate optimal (rescaled) profiles in a large, $n$-dependent box. These optimal profiles are determined by a (deterministic) variational problem.

The topic of the present paper has deep connections to large deviation properties of self-intersections of the walk. This is immediate in the important special case of a standard Gaussian scenery $Y$. Indeed, the conditional distribution of $Z_{n}$ given the random walk $S$ is a centered Gaussian with variance equal to

$$
\Lambda_{n}=\sum_{z \in \mathbb{Z}^{d}} \ell_{n}(z)^{2}=\left\|\ell_{n}\right\|_{2}^{2},
$$

which is often called the self-intersection local time. Hence, large deviations for the random walk in Gaussian scenery would be a consequence of an appropriate large deviation statement for self-intersection local times. However, the latter problem is notoriously difficult and is, up to the best of our knowledge, open in the precision we would need in the present paper. (However, compare to interesting and deep work on self-intersections and mutual intersections by $\mathrm{X}$. Chen [12].) Recent results for self-intersection local times for random walks in dimension $d \geqslant 5$ and applications to random walk in random scenery are given in [3].

The remainder of Section 1 is organized as follows. Our main results are in Section 1.2, a heuristic derivation may be found in Section 1.3, a partial result for Gaussian sceneries for dimension $d=2$ is in Section 1.4. The structure of the remainder of the paper is as follows. In Section 2 we analyze the variational formulas, in Section 3 we present the tools for our proofs of the main results, in Sections 4 and 5 we give the proofs of the upper and the lower bounds, respectively, and finally in Appendix A, we provide the proof of a large deviation principle that is needed in the paper.

\subsection{Results}

Our precise assumptions on the random walk, $S$, are the following. The walker starts at $S_{0}=0$, and the steps have mean zero and some finite exponential moments, more precisely,

$$
\mathbb{E}\left[\mathrm{e}^{t\left|S_{1}\right|}\right]<\infty \text { for some } t>0 .
$$

By $\Gamma \in \mathbb{R}^{d \times d}$ we denote the covariance matrix of the walk's step distribution. Hence, $S$ lies in the domain of attraction of the Brownian motion with covariance matrix $\Gamma$. We assume that $\Gamma$ is a regular matrix. Furthermore, we assume that $S$ is strongly aperiodic, i.e., for any $z \in \mathbb{Z}^{d}$, the smallest subgroup of $\mathbb{Z}^{d}$ that contains $\left\{z+x: \mathbb{P}\left(S_{1}=x\right)>0\right\}$ is $\mathbb{Z}^{d}$ itself. Finally, to avoid technical difficulties, we also assume that the transition function of the walk is symmetric, i.e., $p(0, z)=p(0,-z)$ for $z \in \mathbb{Z}^{d}$, where $p(z, \tilde{z})$ denotes the walker's one-step probability from $z \in \mathbb{Z}^{d}$ to $\tilde{z} \in \mathbb{Z}^{d}$.

Our assumptions on the scenery are the following. Let $Y=(Y(z))_{z \in \mathbb{Z}^{d}}$ be a family of i.i.d. random variables, not necessarily having finite expectation, such that

$$
\mathbb{E}\left[\mathrm{e}^{t Y(0)}\right]<\infty \text { for every } t>0 .
$$

In particular, the cumulant generating function of $Y(0)$, is finite:

$$
H(t)=\log \mathbb{E}\left[\mathrm{e}^{t Y(0)}\right]<\infty, \quad t>0 .
$$

In some of our results, we additionally suppose the following.

Assumption (Y). There are constants $D>0$ and $q>1$ such that

$$
\log \mathbb{P}(Y(0)>r) \sim-D r^{q}, \quad r \rightarrow \infty .
$$

According to Kasahara's exponential Tauberian theorem (see [5, Theorem 4.12.7]), Assumption (Y) is equivalent to

$$
H(t) \sim \widetilde{D} t^{p}, \quad \text { as } t \rightarrow \infty, \text { where } \widetilde{D}=(q-1)\left(D q^{q}\right)^{1 /(1-q)} \text { and } \frac{1}{q}+\frac{1}{p}=1 .
$$


In our first main result, we consider the case of sequences $\left(b_{n}\right)_{n}$ tending to infinity slower than $n^{1 / q}$. By $\nabla$ we denote the usual gradient acting on sufficiently regular functions $\mathbb{R}^{d} \rightarrow \mathbb{R}$. By $H^{1}\left(\mathbb{R}^{d}\right)$ we denote the usual Sobolev space, and we write $\|\nabla \psi\|_{2}^{2}=\int_{\mathbb{R}^{d}}|\nabla \psi(x)|^{2} \mathrm{~d} x$. We use the notation $b_{n} \gg c_{n}$ if $\lim _{n \rightarrow \infty} b_{n} / c_{n}=\infty$.

Theorem 1.1 (Very large deviations). Suppose that Assumption $(\mathrm{Y})$ holds with some $q>\frac{d}{2}$. Pick a sequence $\left(b_{n}\right)_{n \in \mathbb{N}}$ satisfying $1 \ll b_{n} \ll n^{1 / q}$. Then

$$
\lim _{n \rightarrow \infty} n^{-d /(d+2)} b_{n}^{-2 q /(d+2)} \log \mathbb{P}\left(\frac{1}{n} Z_{n}>b_{n}\right)=-K_{D, q},
$$

where

$$
K_{D, q} \equiv \inf \left\{\frac{1}{2}\left\|\Gamma^{1 / 2} \nabla \psi\right\|_{2}^{2}+D\left\|\psi^{2}\right\|_{p}^{-q}: \psi \in H^{1}\left(\mathbb{R}^{d}\right),\|\psi\|_{2}=1\right\},
$$

(we recall that $\frac{1}{p}+\frac{1}{q}=1$ ), and $K_{D, q}$ is positive.

Remark 1.2. For $q \in\left(1, \frac{d}{2}\right)$, (1.9) also holds true, but $K_{D, q}=0$. Indeed, this follows from Proposition 1.6 below together with our proof of Theorem 1.1. One can also see this directly by giving an explicit lower bound for $\log \mathbb{P}\left(\frac{1}{n} Z_{n}>b_{n}\right)$ which runs on a strictly smaller scale than $n^{d /(d+2)} b_{n}^{2 q /(d+2)}$. It remains an open problem in this paper to determine the precise logarithmic rate of $\mathbb{P}\left(\frac{1}{n} Z_{n}>b_{n}\right)$ in the case $q \in\left(1, \frac{d}{2}\right)$. The case $q=\frac{d}{2}$ seems even more delicate and is also left open in the present paper. The case $q \in(0,1)$ has been studied in [16].

Note that the variational problem in (1.10) is of independent interest; it also appeared in [4, Theorem 1.1] in the context of heat kernel asymptotics. In Proposition 1.6 below it turns out that $K_{D, q}$ is positive if and only if $q \geqslant \frac{d}{2}$.

Our next result essentially extends [1, Theorem 2.2] from the case of bounded sceneries to the case in (1.6).

Theorem 1.3 (Large deviations). Suppose that (1.6) holds. Assume that $\mathbb{E}[Y(0)]=0$, and set $\bar{p} \equiv \lim \sup _{t \rightarrow \infty} \frac{\log H(t)}{\log t}$. Assume that $\bar{p}<\infty$ in $d \leqslant 2$ respectively $\bar{p}<\frac{d}{d-2}$ in $d \geqslant 3$. Then, for any $u>0$ satisfying $u \in \operatorname{supp}(Y(0))^{\circ}$,

$$
\lim _{n \rightarrow \infty} n^{-d /(d+2)} \log \mathbb{P}\left(\frac{1}{n} Z_{n}>u\right)=-K_{H}(u),
$$

where

$$
K_{H}(u) \equiv \inf \left\{\frac{1}{2}\left\|\Gamma^{1 / 2} \nabla \psi\right\|_{2}^{2}+\Phi_{H}\left(\psi^{2}, u\right): \psi \in H^{1}\left(\mathbb{R}^{d}\right),\|\psi\|_{2}=1\right\},
$$

and

$$
\Phi_{H}\left(\psi^{2}, u\right)=\sup _{\gamma \in(0, \infty)}\left[\gamma u-\int_{\mathbb{R}^{d}} H\left(\gamma \psi^{2}(y)\right) \mathrm{d} y\right] .
$$

The constant $K_{H}(u)$ is positive.

Switching to the scenery $-Y$, one may, under appropriate conditions, use Theorem 1.3 to obtain the 'other half' of a full large deviation principle for $\left(\frac{1}{n} Z_{n}\right)_{n}$. This was carried out in [1] for bounded sceneries. For Brownian motion in a Gaussian scenery, a result analogous to Theorems 1.1 and 1.3 is [10, Theorem 2].

Note that the constant $K_{H}(u)$ depends on the entire scenery distribution, while $K_{D, q}$ in (1.10) only depends on its upper tails.

Remark 1.4. A statement analogous to Remark 1.2 also applies here: for dimensions $d \geqslant 3$, when $\lim \inf f_{t \rightarrow \infty} \frac{\log H(t)}{\log t}>$ $\frac{d}{d-2},(1.11)$ also holds true, but $K_{H}(u)=0$ for any $u>0$. It was shown recently in [2] that under Assumption (Y) with $q \in\left(1, \frac{d}{2}\right)$ and an additional symmetry assumption, $\log \mathbb{P}\left(\frac{1}{n} Z_{n}>b_{n}\right)$ is of the order $n^{q /(q+2)}$. The case $q \in(0,1)$ has been studied in [16]. 
Remark 1.5 (Large deviations and non-convexity). It is easy to see that, in the special case where $H(t)=\widetilde{D} t^{p}$ (see (1.8)), $K_{H}(u)=u^{2 q /(d+2)} K_{D, q}$, for any $u>0$. (For asymptotic scaling relations see Lemma 1.7.) In particular, $\frac{1}{n} Z_{n}$ satisfies a large deviation principle on $(0, \infty)$ with speed $n^{d /(d+2)}$ and rate function $u \mapsto u^{2 q /(d+2)} K_{D, q}$. This function is strictly convex for $q>\frac{d}{2}+1$ and strictly concave for $q<\frac{d}{2}+1$. In the important special case of a centered Gaussian scenery, Theorem 1.3 contains non-trivial information only in the case $d \in\{1,2,3\}$, in which the rate function is strictly convex, linear and strictly concave, respectively; see also [11] and [10].

The non-convexity around zero for bounded sceneries in $d \in\{3,4\}$ was found in [1] by proving that $K_{H}(u) \geqslant$ $C u^{4 /(d+2)}$ as $u \rightarrow 0$ for some positive constant $C$.

The upper bounds in Theorems 1.1 and 1.3 are proved in Section 4, and the lower bounds in Section 5. We consider only sequences $b_{n} \geqslant 1$ there. The case $a_{n}^{(0)} \ll b_{n} \ll 1$ seems subtle and is left open in the present paper; however see Section 1.4 for a partial result.

Our next proposition gives almost sharp criteria for the positivity of the constants $K_{D, q}$ and $K_{H}(u)$ appearing in Theorems 1.1 and 1.3.

Proposition 1.6 (Positivity of the constants). Fix $d \in \mathbb{N}$ and $p, q>1$ satisfying $\frac{1}{p}+\frac{1}{q}=1$.

(i) For any $D>0$,

$$
K_{D, q}=(d+2)\left(\frac{D}{2}\right)^{2 /(d+2)}\left(\frac{\chi_{d, p}}{d}\right)^{d /(d+2)},
$$

where

$$
\chi_{d, p}=\inf \left\{\frac{1}{2}\left\|\Gamma^{1 / 2} \nabla \psi\right\|_{2}^{2}: \psi \in H^{1}\left(\mathbb{R}^{d}\right):\|\psi\|_{2}=1=\|\psi\|_{2 p}\right\} .
$$

The constant $\chi_{d, p}$ is positive if and only if $d \leqslant \frac{2 p}{p-1}=2 q$. Hence, $K_{D, q}$ is positive if and only if $d \leqslant \frac{2 p}{p-1}=2 q$.

(ii) The constant $K_{H}(u)$ is positive for any $u>\mathbb{E}[Y(0)]=0$ if

$$
\limsup _{t \rightarrow \infty} \frac{\log H(t)}{\log t}< \begin{cases}\infty & \text { if } d \leqslant 2 \\ \frac{d}{d-2} & \text { if } d \geqslant 3 .\end{cases}
$$

For $d \geqslant 3$, if $\liminf _{t \rightarrow \infty} \frac{\log H(t)}{\log t}>\frac{d}{d-2}$, then $K_{H}(u)=0$ for any $u>0$.

The proof of Proposition 1.6 is in Section 2. There we also clarify the relation between $\chi_{d, p}$ and the so-called Gagliardo-Nirenberg constant.

Now we formulate asymptotic relations between the rates obtained in Theorems 1.1 and 1.3.

Lemma 1.7 (Asymptotic scaling relations). Fix $D>0$ and $q>1$, and recall (1.8).

(i) Assume that $H(t) \sim \widetilde{D} t^{p}$ as $t \rightarrow \infty$, then

$$
K_{H}(u) \sim u^{2 q /(d+2)} K_{D, q} \quad \text { as } u \rightarrow \infty .
$$

(ii) Assume that $\mathbb{E}[Y(0)]=0$ and $\mathbb{E}\left[Y(0)^{2}\right]=1$, then

$$
K_{H}(u) \leqslant u^{4 /(d+2)}\left[K_{\frac{1}{2}, 2}+\mathrm{o}(1)\right] \text { as } u \downarrow 0 .
$$

The proof of Lemma 1.7 is in Section 2.4 .

Remark 1.8. We conjecture that the lower bound in (1.17) also holds under an appropriate upper bound on $H$. It is clear (see Remark 1.5 and note the monotonicity of $K_{H}(u)$ in $H$ ) that $u^{-4 /(d+2)} K_{H}(u) \geqslant K_{D, 2}$ for every $u>0$ if $H(t) \leqslant \widetilde{D} t^{2}$ for every $t \geqslant 0$. The positivity of $\liminf _{u \downarrow 0} u^{-4 /(d+2)} K_{H}(u)$ (for cumulant generating functions $H$ of bounded variables) is contained in [1] as part of the proof for non-convexity of the rate function $K_{H}$ in $d \in\{3,4\}$. Since $K_{D, 2}=0$ in $d>4$, it is clear that this proof must fail in $d>4$. 
Lemma 1.7(i) is consistent with Theorems 1.1 and 1.3.

\subsection{Heuristic derivation of Theorems 1.1 and 1.3}

The asymptotics in (1.9) and (1.11) are based on large deviation principles for scaled versions of the walker's local times $\ell_{n}$ and the scenery $Y$. A short summary of the joint optimal strategy of the walker and the scenery is the following. Let us first explain the exponential decay rate of the probabilities under consideration. Assume that $1 \ll b_{n} \ll n^{1 / q}$. In order to contribute optimally to the event $\left\{\frac{1}{n} Z_{n}>b_{n}\right\}$, the walker spreads out over a region whose diameter is of order $\alpha_{n}$ (for a particular choice of $\alpha_{n}$, depending on the sequence $\left.\left(b_{n}\right)_{n}\right)$. The cost for this behavior is $\mathrm{e}^{\mathcal{O}\left(n \alpha_{n}^{-2}\right)}$. The scenery assumes extremely large values within that region, more precisely: values of the order $b_{n}$. The cost for doing that is $\exp \left\{\mathcal{O}\left(b_{n}^{q} \alpha_{n}^{d}\right)\right\}$, under Assumption (Y). The choice of $\alpha_{n}$ is now determined by putting

$$
\frac{n}{\alpha_{n}^{2}}=\alpha_{n}^{d} b_{n}^{q}
$$

A calculation shows that for this choice of $\alpha_{n}$ both sides of (1.18) are equal to the logarithmic decay order of the probability $\mathbb{P}\left(\frac{1}{n} Z_{n}>b_{n}\right)$ in Theorem 1.1 .

Next we give a more precise argument for the very large deviations (Theorem 1.1) which also explains the constants on the right-hand side of (1.9). Introduce the scaled and normalized version of the walker's local times,

$$
L_{n}(x)=\frac{\alpha_{n}^{d}}{n} \ell_{n}\left(\left\lfloor x \alpha_{n}\right\rfloor\right), \quad x \in \mathbb{R}^{d} .
$$

Then $L_{n}$ is a random element of the set

$$
\mathcal{F}=\left\{\psi^{2} \in L^{1}\left(\mathbb{R}^{d}\right):\|\psi\|_{2}=1\right\}
$$

of all Lebesgue probability densities on $\mathbb{R}^{d}$. Furthermore, introduce the scaled version of the field,

$$
\bar{Y}_{n}(x)=\frac{1}{b_{n}} Y\left(\left\lfloor x \alpha_{n}\right\rfloor\right), \quad x \in \mathbb{R}^{d} .
$$

Then we have, writing $\langle\cdot, \cdot\rangle$ for the inner product on $L^{2}\left(\mathbb{R}^{d}\right)$,

$$
\frac{1}{n} Z_{n}=\frac{1}{n} \sum_{z \in \mathbb{Z}^{d}} \frac{n}{\alpha_{n}^{d}} L_{n}\left(\frac{z}{\alpha_{n}}\right) b_{n} \bar{Y}_{n}\left(\frac{z}{\alpha_{n}}\right)=b_{n}\left\langle L_{n}, \bar{Y}_{n}\right\rangle .
$$

Hence, the logarithmic asymptotics of the probability $\mathbb{P}\left(\frac{1}{n} Z_{n}>b_{n}\right)=\mathbb{P}\left(\left\langle L_{n}, \bar{Y}_{n}\right\rangle>1\right)$ will be determined by a combination of large deviation principles for $L_{n}$ and $\bar{Y}_{n}$.

In the spirit of the celebrated large deviation theorem of Donsker and Varadhan, the distributions of $L_{n}$ satisfy a weak large deviation principle in the weak $L^{1}$-topology on $\mathcal{F}$ with speed $n \alpha_{n}^{-2}$ and rate function $\mathcal{I}: \mathcal{F} \rightarrow[0, \infty]$ given by

$$
\mathcal{I}\left(\psi^{2}\right)= \begin{cases}\frac{1}{2}\left\|\Gamma^{1 / 2} \nabla \psi\right\|_{2}^{2} & \text { if } \psi \in H^{1}\left(\mathbb{R}^{d}\right), \\ \infty & \text { otherwise. }\end{cases}
$$

Roughly speaking, this principle says that, for $\psi^{2} \in \mathcal{F}$,

$$
\mathbb{P}\left(L_{n} \approx \psi^{2}\right) \approx \exp \left\{-\frac{n}{\alpha_{n}^{2}} \mathcal{I}\left(\psi^{2}\right)\right\}, \quad n \rightarrow \infty .
$$

Using Assumption (Y), we see that the distributions of $\bar{Y}_{n}$ should satisfy, for any $R>0$, a weak large deviation principle on some appropriate set of sufficiently regular functions $[-R, R]^{d} \rightarrow(0, \infty)$ with speed $\alpha_{n}^{d} b_{n}^{q}$ and rate function

$$
\Phi_{D, q}(\varphi)=D \int_{[-R, R]^{d}} \varphi^{q}(x) \mathrm{d} x,
$$

as the following heuristic calculation suggests: 


$$
\begin{aligned}
\mathbb{P}\left(\bar{Y}_{n} \approx \varphi \text { on }[-R, R]^{d}\right) & \approx \mathbb{P}\left(Y(z)>b_{n} \varphi\left(\frac{z}{\alpha_{n}}\right) \text { for } z \in\left[-R \alpha_{n}, R \alpha_{n}\right]^{d} \cap \mathbb{Z}^{d}\right) \\
& \approx \prod_{z \in\left[-R \alpha_{n}, R \alpha_{n}\right]^{d} \cap \mathbb{Z}^{d}} \exp \left\{-D\left[b_{n} \varphi\left(\frac{z}{\alpha_{n}}\right)\right]^{q}\right\} \\
& \approx \exp \left\{-D \alpha_{n}^{d} b_{n}^{q} \int_{[-R, R]^{d}} \varphi^{q}(x) \mathrm{d} x\right\} .
\end{aligned}
$$

Note that the speeds of the two large deviation principles in (1.24) and (1.25) are equal because of (1.18). Using the two large deviation principles and (1.22), we see that

$$
\mathbb{P}\left(\frac{1}{n} Z_{n}>b_{n}\right) \approx \exp \left\{-\frac{n}{\alpha_{n}^{2}} \widetilde{K}_{D, q}\right\},
$$

where

$$
\widetilde{K}_{D, q}=\inf \left\{\mathcal{I}\left(\psi^{2}\right)+D\|\varphi\|_{q}^{q}: \psi^{2} \in \mathcal{F}, \varphi \in \mathcal{C}_{+}\left(\mathbb{R}^{d}\right),\left\langle\psi^{2}, \varphi\right\rangle=1\right\} .
$$

It is an elementary task to evaluate the infimum on $\varphi$ and to check that indeed $K_{D, q}=\widetilde{K}_{D, q}$. This ends the heuristic explanation of Theorem 1.1.

The situation in the large deviation case, Theorem 1.3, is similar, when we put $b_{n}=1$. See [1] for a heuristic argument in this case.

We distinguish the two cases of very large deviations (V) and large deviations (L). The choices of $b_{n}$ and $\alpha_{n}$ in the respective cases are the following.

$$
\begin{array}{llll}
\text { case (V): } & \text { Hypothesis of Theorem 1.1, } & 1 \ll b_{n} \ll n^{1 / q}, & \alpha_{n}=n^{1 /(d+2)} b_{n}^{-q /(d+2)}, \\
\text { case (L): } & \text { Hypothesis of Theorem 1.3, } & b_{n}=1, & \alpha_{n}=n^{1 /(d+2)} .
\end{array}
$$

\subsection{Small deviations for Gaussian sceneries}

Theorems 1.1 and 1.3 do not handle sequences $\left(b_{n}\right)_{n}$ satisfying $a_{n}^{(0)} \ll b_{n} \ll 1$, where we recall from (1.1) that $a_{n}^{(0)}$ is the scale of the convergence in distribution. In this regime, we present a partial result for Gaussian sceneries and simple random walk in $d=2$. This result is based on a deep result by Brydges and Slade [9] about exponential moments of the renormalized self-intersection local time of simple random walk.

Lemma 1.9 (Small deviations for Gaussian sceneries). Assume that $Y(0)$ is a standard Gaussian random variable and that $\left(S_{n}\right)_{n}$ is the simple random walk, and assume that $d=2$. Let $n^{-1 / 2}(\log n)^{1 / 2}=a_{n}^{(0)} \ll b_{n} \ll a_{n}^{(1)} \equiv n^{-1 / 2} \log n$, then

$$
\lim _{n \rightarrow \infty} \frac{\log n}{b_{n}^{2} n} \log \mathbb{P}\left(\frac{1}{n} Z_{n}>b_{n}\right)=-\frac{\pi}{4}
$$

Proof. As we mentioned in Section 1.1, the distribution of the random walk in random scenery, $Z_{n}$, is easily identified in terms of the walk's self-intersection local time $\Lambda_{n}$ defined in (1.4). More precisely, the conditional distribution of $Z_{n}$ given the walk $S$ is $\mathcal{N} \times \sqrt{\Lambda_{n}}$, where $\mathcal{N}$ is a standard normal variable, independent of the walk. The typical behavior of the self-intersection local time is as follows [9]

$$
\mathbb{E}\left[\Lambda_{n}\right] \sim \frac{2}{\pi}\left(n a_{n}^{(0)}\right)^{2}=\frac{2}{\pi} n \log n, \quad n \rightarrow \infty .
$$

We prove now the upper bound in (1.28). Recall that $d=2$ and introduce the centered and normalized self-intersection local time,

$$
\gamma_{n}=\frac{1}{n}\left(\Lambda_{n}-\mathbb{E}\left[\Lambda_{n}\right]\right)
$$


Use Chebyshev's inequality to obtain, for any $\theta>0$ and any $n \in \mathbb{N}$,

$$
\mathbb{P}\left(\frac{1}{n} Z_{n}>b_{n}\right) \leqslant \mathbb{E}\left[\mathrm{e}^{\theta Z_{n}}\right] \mathrm{e}^{-\theta b_{n} n}
$$

Using the above characterization of the distribution of $Z_{n}$, we see that

$$
\mathbb{E}\left[\mathrm{e}^{\theta Z_{n}}\right]=\mathbb{E}\left[\mathbb{E}\left[\mathrm{e}^{\theta Z_{n}} \mid S\right]\right]=\mathbb{E}\left[\mathbb{E}\left[\exp \left\{\theta \mathcal{N} \sqrt{\Lambda_{n}}\right\} \mid S\right]\right]=\mathbb{E}\left[\mathrm{e}^{\frac{1}{2} \theta^{2} \Lambda_{n}}\right]=\mathbb{E}\left[\mathrm{e}^{\frac{1}{2} \theta^{2} n \gamma_{n}}\right] \mathrm{e}^{\frac{1}{2} \theta^{2} \mathbb{E}\left[\Lambda_{n}\right]}
$$

According to Theorem 1.2 in [9], $\lim _{n \rightarrow \infty} \mathbb{E}\left[\mathrm{e}^{c \gamma_{n}}\right]$ exists and is finite for any $c<c_{0}$, where $c_{0}>0$ is some positive constant. Now pick $\theta=\theta_{n}=\pi b_{n} /(2 \log n)$. Note that $\theta_{n}^{2} n \rightarrow 0$ because of $b_{n} \ll n^{-1 / 2} \log n$, and therefore the first factor on the right-hand side of (1.31) is bounded, according to the above mentioned result of Brydges and Slade. Use (1.29) on the right-hand side of (1.31) and substitute in (1.30) to obtain

$$
\log \mathbb{P}\left(\frac{1}{n} Z_{n}>b_{n}\right) \leqslant-(1+\mathrm{o}(1)) \frac{\pi}{4} \frac{b_{n}^{2} n}{\log n} .
$$

This is the upper bound in (1.28).

Now we prove the lower bound in (1.28). Using the above characterization of the distribution of $Z_{n}$, we obtain, for any $\theta>0$,

$$
\mathbb{P}\left(\frac{1}{n} Z_{n}>b_{n}\right) \geqslant \mathbb{P}(\mathcal{N}>\theta) \mathbb{P}\left(\Lambda_{n}>\frac{n^{2} b_{n}^{2}}{\theta^{2}}\right) .
$$

Fix an arbitrary $c \in\left(0, \frac{2}{\pi}\right)$. We apply (1.32) to $\theta=b_{n}\left(\frac{n}{c \log n}\right)^{1 / 2}$ and obtain

$$
\log \mathbb{P}\left(\frac{1}{n} Z_{n}>b_{n}\right) \geqslant-\frac{1}{2} b_{n}^{2} \frac{n}{c \log n}(1+\mathrm{o}(1))+\log \mathbb{P}\left(\Lambda_{n}>c n \log n\right), \quad n \rightarrow \infty .
$$

By the Paley-Zygmund inequality (Kahane [18] p. 8) stating that $\mathbb{P}(X>r \mathbb{E}[X]) \geqslant(1-r)^{2} \mathbb{E}[X]^{2} / \mathbb{E}\left[X^{2}\right]$ for all $r \in(0,1)$ and all square-integrable random variables $X$, we obtain that

$$
\mathbb{P}\left(\Lambda_{n}>c n \log n\right) \geqslant\left(1-\left(\frac{c \pi}{2}\right)^{2}\right) \frac{\mathbb{E}\left[\Lambda_{n}\right]^{2}}{\mathbb{E}\left[\Lambda_{n}^{2}\right]} .
$$

Recall from (1.29) that $\mathbb{E}\left[\Lambda_{n}\right] \sim \frac{2}{\pi} n \log n$ as $n \rightarrow \infty$. On the other hand, Bolthausen [6] proved that $\operatorname{Var}\left[\Lambda_{n}\right]=\mathcal{O}\left(n^{2}\right)$. Therefore, $\mathbb{E}\left[\Lambda_{n}^{2}\right] \sim \mathbb{E}\left[\Lambda_{n}\right]^{2}$, and, consequently,

$$
\liminf _{n \rightarrow \infty} \mathbb{P}\left(\Lambda_{n}>c n \log n\right)>0 .
$$

Therefore,

$$
\liminf _{n \rightarrow \infty} \frac{\log n}{b_{n}^{2} n} \log \mathbb{P}\left(\frac{1}{n} Z_{n}>b_{n}\right) \geqslant-\frac{1}{2 c} .
$$

Letting $c \uparrow \frac{2}{\pi}$, this yields the lower bound in (1.28).

\section{Variational formulas}

In this section we prove Proposition 1.6 and Lemma 1.7. In Section 2.1 we prove a necessary and sufficient criterion for positivity of the constant $\chi_{d, p}$ defined in (1.15). The relation to the Gagliardo-Nirenberg constant is discussed in Section 2.2, and the relation to the constant $K_{D, q}$ defined in (1.10) is proved in Section 2.3, where we also finish the proof of Proposition 1.6. Finally, Lemma 1.7 is proved in Section 2.4.

\subsection{Positivity of $\chi_{d, p}$}

Lemma 2.1. The constant $\chi_{d, p}$ is positive if and only if $d \leqslant \frac{2 p}{p-1}$. 
Proof. Certainly, it suffices to do the proof only in the case where $\frac{1}{2} \Gamma$ is the identity matrix.

See [12, Section 2] for an alternate proof of the positivity of $\chi_{d, p}$ in the subcritical dimensions, $d<\frac{2 p}{p-1}$, using the relation to the Gagliardo-Nirenberg constant, which we explain in Section 2.2.

Let us recall standard Sobolev inequalities (see [21, Theorems 8.3, 8.5]). There are positive constants $S_{d}$ for $d \geqslant 3$ and $S_{2, r}$ for $r>2$ such that

$$
\begin{aligned}
& S_{d}\|\psi\|_{2 d /(d-2)}^{2} \leqslant\|\nabla \psi\|_{2}^{2}, \quad \text { for } d \geqslant 3, \psi \in D^{1}\left(\mathbb{R}^{d}\right) \cap L^{2}\left(\mathbb{R}^{d}\right), \\
& S_{2, r}\|\psi\|_{r}^{2} \leqslant\|\nabla \psi\|_{2}^{2}+\|\psi\|_{2}^{2}, \quad \text { for } d=2, \psi \in H^{1}\left(\mathbb{R}^{d}\right), r>2 .
\end{aligned}
$$

Here $D^{1}\left(\mathbb{R}^{d}\right)$ denotes the set of locally integrable functions $\mathbb{R}^{d} \rightarrow \mathbb{R}$ which vanish at infinity and possess a distributional derivative in $L^{2}\left(\mathbb{R}^{d}\right)$.

Let us first do the proof for the case $3 \leqslant d \leqslant \frac{2 p}{p-1}$. For any $\psi \in H^{1}\left(\mathbb{R}^{d}\right)$ that satisfies $\|\psi\|_{2}=1=\|\psi\|_{2 p}$, we may use the above Sobolev inequality and obtain that $\|\nabla \psi\|_{2}^{2} \geqslant$ cst. $\|\psi\|_{2 d /(d-2)}^{2}$. We now rewrite

$$
\int_{\mathbb{R}^{d}} \psi^{2 d /(d-2)}(t) \mathrm{d} t=\int_{\mathbb{R}^{d}}\left(\psi^{2 p-2}(t)\right)^{2 /((d-2)(p-1))} \psi^{2}(t) \mathrm{d} t .
$$

Recall that $\psi^{2}$ is a probability density. Therefore, an application of Jensen's inequality to the convex map $x \mapsto$ $x^{2 /[(d-2)(p-1)]}$ yields that $\|\psi\|_{2 d /(d-2)}$ satisfies a lower bound in terms of a power of $\|\psi\|_{2 p}$, which is equal to one. Hence, on the set of those $\psi \in H^{1}\left(\mathbb{R}^{d}\right)$ that satisfy $\|\psi\|_{2}=1=\|\psi\|_{2 p}$, the map $\psi \mapsto\|\nabla \psi\|_{2}^{2}$ is bounded away from zero. Now compare to (1.15) to see that this implies the assertion in the case $3 \leqslant d \leqslant \frac{2 p}{p-1}$.

Now we turn to $d=2$ with $p>1$ arbitrary. By a scaling $\psi_{\beta}=\beta^{d / 2} \psi(\cdot \beta)$, we can find, for any $\delta>0$, a $c(\delta)>0$ such that

$$
\chi_{2, p}=c(\delta) \inf \left\{\|\nabla \psi\|_{2}^{2}: \psi \in H^{1}\left(\mathbb{R}^{d}\right),\|\psi\|_{2}=1,\|\psi\|_{2 p}=\delta\right\} .
$$

Now we choose $\delta$ such that $2 \delta^{-2}=S_{2,2 p}$, the Sobolev constant in (2.1) for $d=2$ and $r=2 p$. Then we have, for any $\psi$ in the set on the right-hand side of (2.2),

$$
2=\frac{2}{\delta^{2}}\|\psi\|_{2 p}^{2}=S_{2,2 p}\|\psi\|_{2 p}^{2} \leqslant\|\nabla \psi\|_{2}^{2}+\|\psi\|_{2}^{2}=\|\nabla \psi\|_{2}^{2}+1,
$$

and hence it follows that $\chi_{2, p} \geqslant c(\delta)>0$.

Now we show that $\chi_{2 d, p} \leqslant 2 \chi_{d, p}$ for any $d \in \mathbb{N}$ and $p \in(0, \infty)$. This simply follows from the observation that, for any $\psi \in H^{1}\left(\mathbb{R}^{d}\right)$, the function $\psi \otimes \psi \in H^{1}\left(\mathbb{R}^{2 d}\right)$ satisfies

$$
\|\nabla(\psi \otimes \psi)\|_{2}^{2}=2\|\nabla \psi\|_{2}^{2} .
$$

Using this, the estimate $\chi_{2 d, p} \leqslant 2 \chi_{d, p}$ easily follows, since $\|\psi \otimes \psi\|_{2}=\|\psi\|_{2}^{2}$ and $\|\psi \otimes \psi\|_{2 p}=\|\psi\|_{2 p}^{2}$. In particular, this shows that $\chi_{1, p}>0$ for any $p>1$.

It remains to show that $\chi_{d, p}=0$ for $d>\frac{2 p}{p-1}$. It is sufficient to construct a sequence of sufficiently regular functions $\psi_{n}: \mathbb{R}^{d} \rightarrow[0, \infty)$ such that $\left\|\psi_{n}\right\|_{2}$ and $\left\|\psi_{n}\right\|_{2 p}$ both converge towards some positive numbers, but $\left\|\nabla \psi_{n}\right\|_{2}$ vanishes as $n \rightarrow \infty$. In order to do this, pick some rotationally invariant function $\psi^{2}=f \circ|\cdot| \in \mathcal{F}$ whose radial part $f:(0, \infty) \rightarrow(0, \infty)$ satisfies

$$
f(r)=D \times \begin{cases}r^{-\gamma} & \text { if } r \in(0,1), \\ 1 & \text { if } r \in[1, A], \\ A^{2 d} r^{-2 d} & \text { if } r>A,\end{cases}
$$

where $A, D, \gamma>0$ are constants to be determined. Let $\omega_{d}$ denote the surface of the unit ball in $\mathbb{R}^{d}$. The following statements can be easily verified by some tedious but elementary calculations:

$$
\begin{aligned}
\gamma<d \quad \Longrightarrow\|\psi\|_{2}^{2}=\frac{\omega_{d}}{d} D\left[2 A^{d}+\frac{\gamma}{d-\gamma}\right]<\infty, \\
\gamma<\frac{d}{p} \quad \Longrightarrow\|\psi\|_{2 p}^{2 p}=\omega_{d} D^{p} \frac{p}{d}\left[\frac{\gamma}{d-p \gamma}+A^{d} \frac{2}{2 p-1}\right]<\infty,
\end{aligned}
$$




$$
\gamma<d-2 \Longrightarrow\|\nabla \psi\|_{2}^{2}=\frac{1}{4} \omega_{d} D\left[\frac{\gamma^{2}}{d-\gamma-2}+A^{d-2} \frac{4 d^{2}}{2+d}\right]<\infty .
$$

Since $p>1$ and $\frac{d}{p}<d-2$, we only have to assume that $\gamma<\frac{d}{p}$. Now we pick sequences $D_{n}, A_{n}$ and $\gamma_{n}$ such that all the following conditions are satisfied as $n \rightarrow \infty$ :

$$
D_{n} \rightarrow 0, \quad A_{n} \rightarrow \infty, \quad \gamma_{n} \uparrow \frac{d}{p}, \quad D_{n} A_{n}^{d} \rightarrow 1, \quad \frac{D_{n}^{p}}{d-p \gamma_{n}} \rightarrow 1 .
$$

Let $\psi_{n}$ be defined as the $\psi$ above with these parameters. Then we have, as $n \rightarrow \infty$,

$$
\left\|\psi_{n}\right\|_{2}^{2} \rightarrow 2 \frac{\omega_{d}}{d}, \quad\left\|\psi_{n}\right\|_{2 p}^{2 p} \rightarrow \omega_{d}, \quad\left\|\nabla \psi_{n}\right\|_{2}^{2} \rightarrow 0 .
$$

This ends the proof.

\subsection{Relation to the Gagliardo-Nirenberg constant}

Actually, for dimensions $d \geqslant 2$ in the special case that $\frac{1}{2} \Gamma$ is the identity matrix, the constant $\chi_{d, p}$ in (1.15) can be identified in terms of the Gagliardo-Nirenberg constant, $\kappa_{d, p}$, as follows. Assume that $d \geqslant 2$ and $1<p<\frac{d}{d-2}$. Then $\kappa_{d, p}$ is defined as the smallest constant $C$ in the Gagliardo-Nirenberg inequality

$$
\|\psi\|_{2 p} \leqslant C\|\nabla \psi\|_{2}^{\frac{d(p-1)}{2 p}}\|\psi\|_{2}^{1-\frac{d(p-1)}{2 p}}, \quad \psi \in H^{1}\left(\mathbb{R}^{d}\right) .
$$

This inequality received a lot of interest from physicists and analysts, and it has deep connections to Nash's inequality and logarithmic Sobolev inequalities. Furthermore, it also plays an important role in recent work of Chen [12] on self-intersections of random walks. See [12, Section 2] for more on the Gagliardo-Nirenberg inequality.

It is clear that

$$
\kappa_{d, p}=\sup _{\psi \in H^{1}\left(\mathbb{R}^{d}\right), \psi \neq 0} \frac{\|\psi\|_{2 p}}{\|\nabla \psi\|_{2}^{\frac{d(p-1)}{2 p}}\|\psi\|_{2}^{1-\frac{d(p-1)}{2 p}}}=\left(\inf _{\psi \in H^{1}\left(\mathbb{R}^{d}\right):\|\psi\|_{2}=1}\|\psi\|_{2 p}^{-\frac{4 q}{d}}\|\nabla \psi\|_{2}^{2}\right)^{-\frac{d}{4 q}} .
$$

Clearly, the term over which the infimum is taken remains unchanged if $\psi$ is replaced by $\psi_{\beta}(\cdot)=\beta^{d / 2} \psi(\cdot \beta)$ for any $\beta>0$. Hence, we can freely add the condition $\|\psi\|_{2 p}=1$ and obtain that $\kappa_{d, p}=\chi_{d, p}^{-d /(4 q)}$. In particular, the variational formulas for $\kappa_{d, p}$ in (2.7) and for $\chi_{d, p}$ in (1.15) have the same maximizer(s) respectively minimizer(s). It is known that (2.7) does possess a maximizer, and this is an infinitely smooth, positive and rotationally invariant function (see [26]). Uniqueness of the minimizer holds in $d \in\{2,3,4\}$ for any $p \in\left(1, \frac{d}{d-2}\right)$, and in $d \in\{5,6,7\}$ for any $p \in\left(1, \frac{8}{d}\right)$, see [22].

\subsection{Relation between $K_{D, q}$ and $\chi_{d, p}$ (Proposition 1.6)}

Now we prove the remaining assertions of Proposition 1.6.

(i) The relation (1.14) is proved by an elementary scaling argument and optimization. Indeed, replace $\psi$ by $\psi_{\beta}(\cdot)=$ $\beta^{d / 2} \psi(\cdot \beta)$ in (1.10) and optimize explicitly on $\beta>0$. Afterwards the additional constraint $\|\psi\|_{2 p}=1$ may freely be added. From (1.14) and Lemma 2.1 the last assertion follows.

(ii) We only show the positivity of $K_{H}(u)$ for $d \geqslant 3$ and $\bar{p}<\frac{d}{d-2}$; the argument for $d \leqslant 2$ and any $\bar{p}>1$ is the same.

Since we assumed that $\mathbb{E}[Y(0)]=0$, we may pick some $\delta>0$ such that $H(t) \leqslant u t / 2$ for $t \in[0, \delta]$. Pick $\varepsilon>0$ such that $\bar{p}+\varepsilon<\frac{d}{d-2}$, then there is $c(\delta, \varepsilon)>0$ depending on $\delta, \varepsilon$ and $H$ only, such that $H(t) \leqslant c(\delta, \varepsilon) t^{\bar{p}+\varepsilon}$ for any $t \in[\delta, \infty)$. Then $H(t) \leqslant \frac{u}{2} t+c(\delta, \varepsilon) t^{\bar{p}+\varepsilon}$ for any $t \geqslant 0$, which implies that, for any $\psi \in H^{1}\left(\mathbb{R}^{d}\right)$ satisfying $\|\psi\|_{2}=1$,

$$
\begin{aligned}
\Phi_{H}\left(\psi^{2}, u\right) & \geqslant \sup _{\gamma>0}\left[\gamma u-\int \frac{u \gamma}{2} \psi^{2}(x) \mathrm{d} x-\int c(\delta, \varepsilon)\left(\gamma \psi^{2}(x)\right)^{\bar{p}+\varepsilon} \mathrm{d} x\right] \\
& =\sup _{\gamma>0}\left[\frac{u}{2} \gamma-c(\delta, \varepsilon) \gamma^{\bar{p}+\varepsilon}\left\|\psi^{2}\right\|_{\bar{p}+\varepsilon}^{\bar{p}+\varepsilon}\right] .
\end{aligned}
$$


Now carry out the optimization over $\gamma$ to see that

$$
\Phi_{H}\left(\psi^{2}, u\right) \geqslant C\left\|\psi^{2}\right\|_{\bar{p}+\varepsilon}^{-q_{\varepsilon}}, \quad \text { where } \frac{1}{\bar{p}+\varepsilon}+\frac{1}{q_{\varepsilon}}=1,
$$

and $C>0$ depends on $u, \bar{p}+\varepsilon$ and $c(\delta, \varepsilon)$ only. Hence, $K_{H}(u) \geqslant K_{C, q_{\varepsilon}}$. Since $d \leqslant 2 q_{\varepsilon}$, this is positive by assertion (i).

Now we show that $K_{H}(u)=0$ for any $u>0$ if $\underline{p} \equiv \liminf _{t \rightarrow \infty} \frac{\log H(t)}{\log t}>\frac{d}{d-2}$. First we do this for a random variable $\widetilde{Y}(0)$ under the assumption that $\mathbb{E}[\tilde{Y}(0)]=1$. Pick $\varepsilon>0$ such that $\underline{p}-\varepsilon>\frac{d}{d-2}$. Since $H^{\prime}(0)=1$, there is $C>0$ such that $H(t) \geqslant C t^{\underline{p}-\varepsilon}$ for any $t \geqslant 0$. Hence, the above argument applies and shows that $K_{H}(u) \leqslant K_{D, q_{\varepsilon}}$ for some $D>0$, where $q_{\varepsilon}$ is determined by $\frac{1}{\underline{p}-\varepsilon}+\frac{1}{q_{\varepsilon}}=1$. Since $\underline{p}-\varepsilon>\frac{d}{d-2}$, the condition $d \leqslant \frac{2(\underline{p}-\varepsilon)}{\underline{p}-\varepsilon-1}$ is violated. Again assertion (i) implies that $K_{H}(u)=0$.

Let now $Y(0)$ have expectation 0 , then $\widetilde{Y}(0)=Y(0)+1$ has expectation 1 . If $\widetilde{H}$ denotes the cumulant generating function of $\widetilde{Y}(0)$, then we have, according to the above, $K_{\widetilde{H}}(u)=0$ for any $u>0$. Since $K_{\widetilde{H}}(u)$ is well-defined, non-negative and non-decreasing for all $u \in \mathbb{R}$, we also have $K_{\widetilde{H}}(u)=0$ for any $u \in \mathbb{R}$. Obviously, $\widetilde{H}(t)=H(t)+t$ and $K_{\widetilde{H}}(u)=K_{H}(u-1)$ for any $u \in \mathbb{R}$, and this implies the statement.

\subsection{Scaling relations (Lemma 1.7)}

In this section, we prove Lemma 1.7 .

(i) Fix $\varepsilon>0$, then there is some $C>0$ such that

$$
-C t+(\widetilde{D}-\varepsilon) t^{p} \leqslant H(t) \leqslant C t+(\widetilde{D}+\varepsilon) t^{p}, \quad t \geqslant 0 .
$$

Using this in the definition of $\Phi_{H}\left(\psi^{2}, u\right)$, we obtain, for any $\psi \in H^{1}\left(\mathbb{R}^{d}\right)$,

$$
\sup _{\gamma>0}\left\{\gamma(u-C)-\gamma^{p}(\widetilde{D}+\varepsilon)\left\|\psi^{2}\right\|_{p}^{p}\right\} \leqslant \Phi_{H}\left(\psi^{2}, u\right) \leqslant \sup _{\gamma>0}\left\{\gamma(u+C)-\gamma^{p}(\widetilde{D}-\varepsilon)\left\|\psi^{2}\right\|_{p}^{p}\right\} .
$$

The suprema may easily be evaluated, and we obtain, for some $\eta_{1}, \eta_{2}>0$, which vanish as $\varepsilon \downarrow 0$,

$$
\left(D-\eta_{1}\right)\left\|\psi^{2}\right\|_{p}^{-q}(u-C)^{q} \leqslant \Phi_{H}\left(\psi^{2}, u\right) \leqslant\left(D+\eta_{2}\right)\left\|\psi^{2}\right\|_{p}^{-q}(u+C)^{q} .
$$

Using this in the definition of $K_{H}(u)$ in (1.12), we obtain

$$
K_{\left(D-\eta_{1}\right)(u-C)^{q}, q} \leqslant K_{H}(u) \leqslant K_{\left(D+\eta_{2}\right)(u+C)^{q}, q} .
$$

Now use Proposition 1.6(i), in particular (1.14), and use that $\eta_{1}, \eta_{2} \rightarrow 0$ as $\varepsilon \downarrow 0$.

(ii) Substituting $\psi(\cdot)=u^{d /(d+2)} \psi_{0}\left(\cdot u^{2 /(d+2)}\right)$ and $\gamma=u^{(2-d) /(2+d)} \gamma_{0}$ yields that

$$
u^{-4 /(d+2)} K_{H}(u)=\inf _{\left\|\psi_{0}\right\|_{2}=1}\left\{\frac{1}{2}\left\|\Gamma^{1 / 2} \nabla \psi_{0}\right\|_{2}^{2}+\sup _{\gamma_{0}>0}\left(\gamma_{0}-\int u^{-2} H\left(u \gamma_{0} \psi_{0}^{2}(x)\right) \mathrm{d} x\right)\right\} .
$$

It remains to show that the limit superior of the right-hand side as $u \downarrow 0$ is not larger than $K_{\frac{1}{2}, 2}$. This is shown as follows. Let $\psi_{*} \in H^{1}\left(\mathbb{R}^{d}\right)$ be an $L^{2}$-normalized bounded minimizer in the variational formula in (1.10) for $D=\frac{1}{2}$ and $q=2$. Its existence is proven in the same way as in [26], where the case $\Gamma=\mathrm{Id}$ was considered. Hence we have $\lim _{u \downarrow 0} \int u^{-2} H\left(u \gamma_{0} \psi_{*}^{2}(x)\right) \mathrm{d} x=\frac{1}{2} \gamma_{0}^{2}\left\|\psi_{*}^{2}\right\|_{2}^{2}$, uniformly in $\gamma_{0}$ on compacts of $[0, \infty)$. Hence, the supremum on the right-hand side of (2.8) converges towards $\sup _{\gamma_{0}>0}\left(\gamma_{0}-\frac{1}{2} \gamma_{0}^{2}\left\|\psi_{*}^{2}\right\|_{2}^{2}\right)=\frac{1}{2}\left\|\psi_{*}^{2}\right\|_{2}^{-2}$. Replacing on the right-hand side of (2.8) the infimum on $\psi_{0}$ by $\psi_{*}$, we arrive at $\limsup _{u \downarrow 0} u^{-4 /(d+2)} K_{H}(u) \leqslant K_{\frac{1}{2}, 2}$, which is (1.17).

\section{Proof of Theorems 1.1 and 1.3: Preparations}

In this section we prepare for the proofs of our main results, Theorems 1.1 and 1.3. Our proofs follow the strategy of the proof of [1, Theorem 2.2]. That is, the proofs of the lower bounds essentially follow the outline described in Section 1.3, and the proofs of the upper bounds use an exponential Chebyshev inequality with a random parameter. However, due to the unboundedness of the scenery in our case, we face a serious additional difficulty, which we will overcome using a recently developed technique. 
As we have already indicated in Section 1.3, our main tools are large deviation principles for the walker's local times and for the scenery. These principles are presented in Sections 3.1 and 3.2, respectively. However, for the application of these two principles, there are three main technical obstacles:

(1) the principles hold only on compact subsets of the space,

(2) the scaled scenery must be smoothed,

(3) the scaled scenery must be cut down to bounded size.

The first obstacle will be handled later by making a connection to the periodized version of the random walk, which is a standard recipe. Hence, it will be necessary to approximate the variational formulas appearing in our main results by finite-space versions, and this is carried out in Section 3.5. The necessity of the smoothing arises from the fact that the map $\left(\psi^{2}, \varphi\right) \mapsto\left\langle\psi^{2}, \varphi\right\rangle$ is not continuous in the product of the topologies on which the large deviation principles are based. This was already pointed out in [1]. The remedy is a smoothing procedure which was introduced in [1] and will be adapted in Section 3.4 below. However, this procedure only works for uniformly bounded sceneries, and this explains the necessity of a cutting argument for the scenery. This obstacle was not present in [1] and is the main technical challenge in the present paper, see Section 3.3.

\subsection{Large deviations for the local times}

In this section, we formulate one of our main tools: large deviation principles for the normalized and scaled local times. These principles are essentially standard and well-known, however, some of the principles we use do not seem to have been proven in the literature, and therefore we shall provide a proof for them in Appendix A.

For the convenience of the reader, we recall the notion of a large deviation principle. A sequence $\left(X_{n}\right)_{n \in \mathbb{N}}$ of random variables (or their distributions), taking values in a topological space $\mathcal{X}$, satisfy a large deviation principle with speed $\left(\gamma_{n}\right)_{n \in \mathbb{N}}$ and rate function $\mathcal{I}: \mathcal{X} \rightarrow[0, \infty]$, if the following two statements hold:

$$
\begin{array}{ll}
\limsup _{n \rightarrow \infty} \frac{1}{\gamma_{n}} \log P\left(X_{n} \in F\right) \leqslant-\inf _{F} \mathcal{I}, \quad F \subset \mathcal{X} \text { closed, } \\
\liminf _{n \rightarrow \infty} \frac{1}{\gamma_{n}} \log P\left(X_{n} \in O\right) \geqslant-\inf _{O} \mathcal{I}, \quad O \subset \mathcal{X} \text { open. }
\end{array}
$$

This definition equally applies if the measure $P$ has not full mass, but happens to be a subprobability measure only.

We shall need large deviation principles for a rescaled version of the local times of our random walk. More precisely, we shall need two slightly different principles: one on never leaving a given cube in $\mathbb{Z}^{d}$ and $\mathbb{R}^{d}$, respectively, and another one for the periodized version of the walk on that cube. We recall that we have listed our assumptions on the random walk at the beginning of Section 1.2. For $R>0$, we denote by $B_{R}=[-R, R]^{d} \cap \mathbb{Z}^{d}$ the centered box in $\mathbb{Z}^{d}$ with radius $R$. By $S^{(R)}=\left(S_{0}^{(R)}, S_{1}^{(R)}, \ldots\right)$ we denote the random walk on the torus $B_{R}$, i.e., the walk on $B_{R}$ (with the opposite sides identified with each other) having transition kernel

$$
p^{(R)}(z, \tilde{z})=\sum_{k \in \mathbb{Z}^{d}} p(z, \tilde{z}+2 k\lfloor R\rfloor), \quad z, \tilde{z} \in B_{R},
$$

where $p(\cdot, \cdot)$ denotes the transition kernel of $S$. Note that $p^{(R)}$ is symmetric since $p$ is. The local times of $S^{(R)}$ are denoted by

$$
\ell_{n}^{(R)}(z)=\sum_{k \in \mathbb{Z}^{d}} \ell_{n}(z+2 k\lfloor R\rfloor), \quad z \in B_{R}
$$

We consider rescaled versions of $\frac{1}{n} \ell_{n}$ and $\frac{1}{n} \ell_{n}^{(R)}$. Recall the normalized and rescaled version $L_{n}$ of the local times $\ell_{n}$ defined in (1.19). By $\mathcal{F}_{R}$ we denote the subset of those functions in $\mathcal{F}$ whose support lies in $Q_{R}=[-R, R]^{d}$. Note that

$$
\operatorname{supp}\left(L_{n}\right) \subset Q_{R} \Longleftrightarrow \operatorname{supp}\left(\ell_{n}\right) \subset B_{R \alpha_{n}} .
$$


Denote the scaled version of the torus-version of the local times, $\frac{1}{n} \ell_{n}^{\left(R \alpha_{n}\right)}$, by $L_{n}^{(R)}: Q_{R} \rightarrow[0, \infty)$. Then $L_{n}^{(R)}$ is a random element of the set $\mathcal{F}^{(R)}$ of probability densities on the torus $Q_{R}=[-R, R]^{d}$, whose opposite sides are identified with each other. We define a rate function $\mathcal{I}^{(R)}: \mathcal{F}^{(R)} \rightarrow[0, \infty]$ by

$$
\mathcal{I}^{(R)}\left(\psi^{2}\right)=\frac{1}{2} \int_{Q_{R}}\left|\Gamma^{1 / 2} \nabla_{R} \psi(x)\right|^{2} \mathrm{~d} x,
$$

if $\psi$ has an extension to an element of $H^{1}\left(\mathbb{R}^{d}\right)$, and $\mathcal{I}^{(R)}\left(\psi^{2}\right)=\infty$ otherwise. Here $\nabla_{R}$ denotes the gradient on the torus $Q_{R}$, i.e., with periodic boundary condition.

The topology used on the sets $\mathcal{F}_{R}$ and on $\mathcal{F}^{(R)}$ are the weak topologies induced by the test integrals against the continuous bounded functions on $Q_{R}$. If we identify any element of $\mathcal{F}_{R}$ resp. of $\mathcal{F}^{(R)}$ with a probability measure, then this topology is just the usual weak topology on the set of probability measures on $Q_{R}$. In this case, we extend the respective rate functions trivially by $\infty$ to the set of measures not having a density.

Lemma 3.1 (Large deviation principles for $L_{n}$ ). Fix $R>0$. Assume that $\alpha_{n} \rightarrow \infty$ and

$$
\alpha_{n}^{d} \ll \begin{cases}\sqrt{n} & \text { if } d=1, \\ \frac{n}{\log n} & \text { if } d=2, \\ n & \text { if } d \geqslant 3,\end{cases}
$$

as $n \rightarrow \infty$. Then the following two facts hold true.

(i) The distributions of $L_{n}$ under $\mathbb{P}\left(\cdot \cap\left\{\operatorname{supp}\left(L_{n}\right) \subset Q_{R}\right\}\right)$ satisfy a large deviation principle on $\mathcal{F}_{R}$ with speed $n \alpha_{n}^{-2}$ and rate function $\mathcal{I}_{R}$, the restriction of $\mathcal{I}$ defined in (1.23) to $\mathcal{F}_{R}$.

(ii) The distributions of $L_{n}^{(R)}$ under $\mathbb{P}$ satisfy a large deviation principle on $\mathcal{F}^{(R)}$ with speed $n \alpha_{n}^{-2}$ and rate function $\mathcal{I}^{(R)}$ given in (3.6).

The upper bound (3.1) of the principle in (i) for the special case of simple random walk and $\alpha_{n}=n^{\frac{1}{d+2}}$ has been proven by Donsker and Varadhan [15], Section 3. We have deferred the proof of Lemma 3.1 to Appendix A. We feel that the statement and its proof are standard and should be known to the experts, but we could not find a reference in the literature. Our proof basically follows the route of [17], which has become standard by now. The strategy for the proof of (i) can be roughly summarized as follows (the proof of (ii) is analogous). We shall identify the cumulant generating function of $L_{n}$ (i.e., the logarithmic asymptotics of exponential moments of test integrals against continuous and bounded functions $f$ ) in terms of the Dirichlet eigenvalue of the operator $\frac{1}{2} \nabla \cdot \Gamma \nabla+f$. In a second step, we prove the large deviation principle via what is called now the abstract Gärtner-Ellis theorem and identify the rate function of the large deviation principle as the Legendre transform of the eigenvalue.

\subsection{Large deviations for the scenery}

In the proofs of the lower bounds in Theorems 1.1 and 1.3, we shall rely on precise large deviation lower bounds for the scenery, tested against fixed functions. The precise formulations are given for the respective cases here. Recall from (1.27) the two cases (V) and (L), which correspond to Theorems 1.1 and 1.3, respectively.

We begin, in case $(\mathrm{V})$ with a large deviation principle for the rescaled scenery $\bar{Y}_{n}$ defined in (1.21).

Lemma 3.2. Assume the case (V) in (1.27), and pick sequences $\left(b_{n}\right)_{n}$ and $\left(\alpha_{n}\right)_{n}$ as in (1.27). Fix $R>0$ and a continuous function $\varphi: Q_{R} \rightarrow(0, \infty)$. Then

$$
\liminf _{n \rightarrow \infty} \frac{1}{\alpha_{n}^{d} b_{n}^{q}} \log \mathbb{P}\left(\bar{Y}_{n} \geqslant \varphi \text { on } Q_{R}\right) \geqslant-D\|\varphi\|_{q}^{q} .
$$

Proof. Fix some small $\varepsilon>0$. It is easy to see that, for sufficiently large $n \in \mathbb{N}$,

$$
\mathbb{P}\left(\bar{Y}_{n} \geqslant \varphi \text { on } Q_{R}\right)=\prod_{z \in B_{R \alpha_{n}}} \mathbb{P}\left(Y(z) \geqslant b_{n} \varphi\left(\frac{z}{\alpha_{n}}\right)\right)
$$




$$
\begin{aligned}
& \geqslant \exp \left\{-(D-\varepsilon) b_{n}^{q} \sum_{z \in B_{R \alpha_{n}}} \varphi\left(\frac{z}{\alpha_{n}}\right)^{q}\right\} \\
& \geqslant \exp \left\{-(D-2 \varepsilon) \alpha_{n}^{d} b_{n}^{q}\|\varphi\|_{q}^{q}\right\} .
\end{aligned}
$$

Let us now proceed with case (L).

Lemma 3.3. Assume the case (L) in (1.27) and fix $R>0, M>0$ and a positive continuous function $\psi^{2}: Q_{R} \rightarrow$ $(0, \infty)$. Recall that $\alpha_{n}=n^{1 /(d+2)}$. Let $\widetilde{H}_{M}$ be the conditional cumulant generating function of $Y(0)$ given that $Y(0) \geqslant-M$. Then, for any $u>0$,

$$
\liminf _{n \rightarrow \infty} \frac{1}{\alpha_{n}^{d}} \log \mathbb{P}\left(\int_{Q_{R}} \bar{Y}_{n}(x) \psi^{2}(x) \mathrm{d} x \geqslant u \mid Y(z) \geqslant-M \forall z \in B_{R \alpha_{n}}\right) \geqslant \Phi_{\widetilde{H}_{M}}\left(\psi^{2}, u ; R\right),
$$

where

$$
\Phi_{H}\left(\psi^{2}, u ; R\right)=\sup _{\gamma>0}\left(\gamma u-\int_{Q_{R}} H\left(\gamma \psi^{2}(x)\right) \mathrm{d} x\right)
$$

is the $Q_{R}$-version of $\Phi_{H}$ defined in (1.13).

Proof. For any $\gamma>0$, we have

$$
\begin{aligned}
\mathbb{E}\left[\exp \left\{\gamma \alpha_{n}^{d} \int_{Q_{R}} \bar{Y}_{n}(x) \psi^{2}(x) \mathrm{d} x\right\} \mid Y(z) \geqslant-M \forall z \in B_{R \alpha_{n}}\right] \\
=\mathbb{E}\left[\exp \left\{\gamma \sum_{z \in B_{R \alpha_{n}}} Y(z) \alpha_{n}^{d} \int_{z / \alpha_{n}+\left[0,1 / \alpha_{n}\right]^{d}} \psi^{2}(x) \mathrm{d} x\right\} \mid Y(z) \geqslant-M \forall z \in B_{R \alpha_{n}}\right] \\
=\prod_{z \in B_{R \alpha_{n}}} \mathrm{e}^{(1+\mathrm{o}(1)) \widetilde{H}_{M}\left(\gamma \psi^{2}\left(z / \alpha_{n}\right)\right)} \\
=\exp \left\{\alpha_{n}^{d} \int_{Q_{R}} \widetilde{H}_{M}\left(\gamma \psi^{2}(x)\right) \mathrm{d} x(1+\mathrm{o}(1))\right\} .
\end{aligned}
$$

According to a variant of the Gärtner-Ellis theorem, $\int_{Q_{R}} \bar{Y}_{n}(x) \psi^{2}(x) \mathrm{d} x$ satisfies, under conditioning on $Y(z) \geqslant-M$ for all $z \in B_{R \alpha_{n}}$, a large deviation principle on $(0, \infty)$ with speed $\alpha_{n}^{d}$ and rate given by the Legendre transform of the map $\gamma \mapsto \int_{Q_{R}} \widetilde{H}_{M}\left(\gamma \psi^{2}(x)\right) \mathrm{d} x$. This transform is equal to the map $u \mapsto \Phi_{\widetilde{H}_{M}}\left(\psi^{2}, u ; R\right)$.

\subsection{The cutting argument}

In this section we provide the cutting argument for the scenery in the cases (V) and (L). Our method consists of a careful analysis of the $k$-th moments of the random walk in random scenery, where $k=k_{n}$ is chosen in an appropriate dependence of $n$. Variants of this method have recently been developed in the study of mutual intersections of random paths in [12] and [20].

Fix sequences $\left(b_{n}\right)_{n}$ and $\left(\alpha_{n}\right)_{n}$ as in (1.27) and consider the scaled normalized scenery $\bar{Y}_{n}$ as defined in (1.21). Fix $M>0$. We use the notation

$$
y^{(\leqslant M)}=(y \wedge M) \vee(-M) \text { and } y^{(>M)}=(y-M)_{+}, \quad \text { for any } y \in \mathbb{R} .
$$

Later we shall estimate the scaled scenery $\bar{Y}_{n}$ by $\bar{Y}_{n} \leqslant \bar{Y}_{n}^{(\leqslant M)}+\bar{Y}_{n}^{(>M)}$. Here we show how we shall handle the second term. 
Proposition 3.4 (Scenery cutting). Assume one of the cases (V) or (L) in (1.27). Then, for any $\varepsilon>0$,

$$
\lim _{M \rightarrow \infty} \limsup _{n \rightarrow \infty} \frac{\alpha_{n}^{2}}{n} \log \mathbb{P}\left(\left\langle L_{n}, \bar{Y}_{n}^{(>M)}\right\rangle>\varepsilon\right)=-\infty .
$$

Proof. Step 1. It suffices to establish that there exists $C_{M}>0$ satisfying $\lim _{M \rightarrow \infty} C_{M}=0$ and

$$
\mathbb{E}\left[\left\langle\ell_{n}, Y^{\left(>M b_{n}\right)}\right\rangle^{k}\right] \leqslant n^{k} b_{n}^{k} C_{M}^{k}, \quad n \in \mathbb{N}, \text { where } k=\frac{n}{\alpha_{n}^{2}} .
$$

Proof. Use the Markov inequality to estimate, for any $\varepsilon, M>0$ and $n, k \in \mathbb{N}$,

$$
\mathbb{P}\left(\left\langle L_{n}, \bar{Y}_{n}^{(>M)}\right\rangle>\varepsilon\right) \leqslant \varepsilon^{-k} \mathbb{E}\left[\left\langle L_{n}, \bar{Y}_{n}^{(>M)}\right\rangle^{k}\right]=\varepsilon^{-k}\left(n b_{n}\right)^{-k} \mathbb{E}\left[\left\langle\ell_{n}, Y^{\left(>M b_{n}\right)}\right\rangle^{k}\right] .
$$

Now put $k=n \alpha_{n}^{-2}$ and observe that the estimate in (3.12) for some $C_{M} \rightarrow 0$ as $M \rightarrow \infty$ implies Proposition 3.4.

Our next step is a variant of the well-known periodization technique which projects the random walk in random scenery into a fixed box. Recall from (3.4) the local times of the periodized random walk.

Step 2. (Periodization) For any $R, n, k \in \mathbb{N}$ and for any i.i.d. scenery $Y$ which is independent of the random walk,

$$
\mathbb{E}\left[\left\langle\ell_{n}, Y\right\rangle^{k}\right] \leqslant \sum_{z_{1}, \ldots, z_{k} \in B_{R}} \mathbb{E}\left[\prod_{i=1}^{k} \ell_{n}^{(R)}\left(z_{i}\right)\right] \prod_{x \in B_{R}} \mathbb{E}\left[|Y(0)|^{\#\left\{i: z_{i}=x\right\}}\right] .
$$

Proof. We write out

$$
\mathbb{E}\left[\left\langle\ell_{n}, Y\right\rangle^{k}\right]=\sum_{z_{1}, \ldots, z_{k} \in B_{R}} \sum_{m_{1}, \ldots, m_{k} \in \mathbb{Z}^{d}} \mathbb{E}\left[\prod_{i=1}^{k} \ell_{n}\left(z_{i}+2 R m_{i}\right)\right] \mathbb{E}\left[\prod_{i=1}^{k} Y\left(z_{i}+2 R m_{i}\right)\right] .
$$

We use that the scenery is i.i.d. and derive, with the help of Jensen's inequality, the estimate

$$
\begin{aligned}
\mathbb{E}\left[\prod_{i=1}^{k} Y\left(z_{i}+2 R m_{i}\right)\right] & =\prod_{x \in B_{R}} \prod_{y \in \mathbb{Z}^{d}} \mathbb{E}\left[Y(y)^{\#\left\{i: z_{i}=x, z_{i}+2 R m_{i}=y\right\}}\right] \\
& \leqslant \prod_{x \in B_{R}} \prod_{y \in \mathbb{Z}^{d}} \mathbb{E}\left[|Y(0)|^{\#\left\{i: z_{i}=x\right\}}\right]^{\frac{\#\left\{i: z_{i}=x, z_{i}+2 R m_{i}=y\right\}}{\#\left\{i: z_{i}=x\right\}}} \\
& =\prod_{x \in B_{R}} \mathbb{E}\left[|Y(0)|^{\#\left\{i: z_{i}=x\right\}}\right] .
\end{aligned}
$$

Use this in (3.14) and carry out the sum over $m_{1}, \ldots, m_{k}$ to finish.

In the next step we estimate the term in (3.13) that involves the walker's local times. We denote by $S^{(R)}$ the periodized version of the random walk in $B_{R}$ and by $p_{s}^{(R)}(x, y)$ its transition probability from $x$ to $y$ in $s$ steps. By

$$
G_{\lambda}^{(R)}(x, y)=\sum_{s=0}^{\infty} \mathrm{e}^{-\lambda s} p_{s}^{(R)}(x, y)
$$

we denote the Green's function associated with the periodized walk, geometrically stopped with parameter $\lambda>0$. $\mathfrak{S}_{k}$ denotes the set of permutations of $1, \ldots, k$.

Step 3. Fix $R>0, \lambda>0$ and $k \in \mathbb{N}$. Then, for any $n \in \mathbb{N}$, and for any $z_{1}, \ldots, z_{k} \in B_{R}$,

$$
\mathbb{E}\left[\prod_{i=1}^{k} \ell_{n}^{(R)}\left(z_{i}\right)\right] \leqslant \mathrm{e}^{\lambda n} \sum_{\sigma \in \mathfrak{S}_{k}} \prod_{i=1}^{k} G_{\lambda}^{(R)}\left(z_{\sigma(i-1)}, z_{\sigma(i)}\right) .
$$

Proof. Writing out the local times, we obtain 


$$
\begin{aligned}
\mathbb{E}\left[\prod_{i=1}^{k} \ell_{n}^{(R)}\left(z_{i}\right)\right] & \leqslant \sum_{t_{1}, \ldots, t_{k}=0}^{n} \mathbb{P}\left(S_{t_{i}}^{(R)}=z_{i}, i=1, \ldots, k\right) \\
& \leqslant \sum_{0 \leqslant t_{1} \leqslant t_{2} \leqslant \ldots \leqslant t_{k} \leqslant n} \sum_{\sigma \in \mathfrak{S}_{k}} \mathbb{P}\left(S_{t_{\sigma(i)}}^{(R)}=z_{i}, i=1, \ldots, k\right) \\
& =\sum_{\sigma \in \mathfrak{S}_{k}} \sum_{s_{1}, \ldots, s_{k} \in \mathbb{N}_{0}} \mathbb{1}\left\{\sum_{i=1}^{k} s_{i} \leqslant n\right\} \prod_{i=1}^{k} p_{s_{i}}^{(R)}\left(z_{\sigma(i-1)}, z_{\sigma(i)}\right),
\end{aligned}
$$

where in the last line we substituted $s_{i}=t_{i}-t_{i-1}$ and wrote $\sigma^{-1}$ instead of $\sigma$. We put $\sigma(0)=0$ and $z_{0}=0$. Now we estimate the indicator by

$$
\mathbb{1}\left\{\sum_{i=1}^{k} s_{i} \leqslant n\right\} \leqslant \mathrm{e}^{\lambda n} \prod_{i=1}^{k} \mathrm{e}^{-s_{i} \lambda} .
$$

Using this in (3.17) and carrying out the sums over $s_{1}, \ldots, s_{k}$, we arrive at the assertion.

In order to further estimate the Greenian term on the right of (3.16), we shall later need the following.

Step 4. Fix $R>0$ and $p^{\prime} \in\left(1, \frac{d}{d-2}\right)$, if $d \geqslant 3$, or $p^{\prime}>1$ if $d \in\{1,2\}$. Then there is a constant $C>0$ such that, for any $n \in \mathbb{N}$ and any $x \in B_{R \alpha_{n}}$,

$$
\sum_{y \in B_{R \alpha_{n}}} G_{\alpha_{n}^{-2}}^{\left(R \alpha_{n}\right)}(x, y)^{p^{\prime}} \leqslant C \alpha_{n}^{d+(2-d) p^{\prime}} .
$$

Proof. For $d \leqslant 4$, we estimate, with the help of Jensen's inequality, and using that $p_{s}^{\left(R \alpha_{n}\right)}(x, y)$ is not bigger than one and that its sum on $y \in B_{R \alpha_{n}}$ equals one,

$$
\begin{aligned}
\sum_{y \in B_{R \alpha_{n}}} G_{\alpha_{n}^{-2}}^{\left(R \alpha_{n}\right)}(x, y)^{p^{\prime}} & =\sum_{y \in B_{R \alpha_{n}}}\left(\sum_{s=0}^{\infty} \mathrm{e}^{-s \alpha_{n}^{-2}} p_{s}^{\left(R \alpha_{n}\right)}(x, y)\right)^{p^{\prime}} \\
& \leqslant\left(1-\mathrm{e}^{-\alpha_{n}^{-2}}\right)^{p^{\prime}-1} \sum_{y \in B_{R \alpha_{n}}} \sum_{s=0}^{\infty} \mathrm{e}^{-s \alpha_{n}^{-2}} p_{s}^{\left(R \alpha_{n}\right)}(x, y) \\
& \leqslant\left(1-\mathrm{e}^{-\alpha_{n}^{-2}}\right)^{p^{\prime}-2} \sim \alpha_{n}^{4-2 p^{\prime}} .
\end{aligned}
$$

Now noting that $4-2 p^{\prime} \leqslant d+(2-d) p^{\prime}$ for $d \leqslant 4$ finishes the proof of (3.18).

For $d \geqslant 4$, we use another argument, which is based on the estimate [25, Theorem 2] $G(0, y) \leqslant C|y|^{2-d}$ for any $y \in \mathbb{Z}^{d} \backslash\{0\}$, where $G$ is the Green's function for the free (i.e., non-stopped and non-periodized) random walk, and $C>0$ is constant. Certainly, it suffices to take $x=0$. We use $C>0$ and $c>0$ to denote generic positive constants, not depending on $n$ or $y$, which may change their values from line to line. We estimate

$$
G_{\alpha_{n}^{-2}}^{\left(R \alpha_{n}\right)}(0, y) \leqslant G(0, y)+\sum_{m \in \mathbb{Z}^{d} \backslash\{0\}} \sum_{s \in \mathbb{N}_{0}} \mathrm{e}^{-s \alpha_{n}^{-2}} p_{s}\left(0, y+2 m R \alpha_{n}\right) .
$$

For the first term, use the above mentioned result to see that $\sum_{y \in B_{R \alpha_{n}}} G(0, y)^{p^{\prime}} \leqslant C \alpha_{n}^{d+(2-d) p^{\prime}}$. With $\gamma>0$ a small auxiliary parameter, we split the sum on $s$ in the parts where $s \leqslant \gamma|m| \alpha_{n}$ and the remainder. Recall that the walker's steps have some exponential moments, see (1.5). Hence, we can estimate, if $\gamma$ is small enough $\left(\gamma<\frac{R}{4} / \log \mathbb{E}\left[\mathrm{e}^{\left|S_{1}\right|}\right]\right.$ suffices), for $|m| \geqslant 1$ and $s \leqslant \gamma|m| \alpha_{n}$, and all $y \in B_{R \alpha_{n}}$,

$$
\begin{aligned}
p_{s}\left(0, y+2 m R \alpha_{n}\right) & \leqslant \mathbb{P}\left(\left|S_{s}\right| \geqslant\left|y+2 m R \alpha_{n}\right|\right) \leqslant \mathbb{E}\left[\mathrm{e}^{\left|S_{1}\right|}\right]^{s} \mathrm{e}^{-\left|y+2 m R \alpha_{n}\right|} \leqslant \mathbb{E}\left[\mathrm{e}^{\left|S_{1}\right|}\right]^{s} \mathrm{e}^{-R \alpha_{n}|m|} \\
& \leqslant \mathrm{e}^{-c|m| \alpha_{n}} .
\end{aligned}
$$


This gives, for any $y \in B_{R \alpha_{n}}$,

$$
\sum_{m \in \mathbb{Z}^{d} \backslash\{0\}} \sum_{s \in \mathbb{N}_{0}: s \leqslant \gamma \alpha_{n}|m|} \mathrm{e}^{-s \alpha_{n}^{-2}} p_{s}\left(0, y+2 m R \alpha_{n}\right) \leqslant C \sum_{m \in \mathbb{Z}^{d} \backslash\{0\}} \mathrm{e}^{-c|m| \alpha_{n}}=\mathrm{o}\left(\alpha_{n}^{2-d}\right) .
$$

The remainder is estimated as follows. We use the local central limit theorem (see [23, Chapter VII, Theorem 13]) to deduce that there are $C>0$ and $c>0$ such that

$$
p_{s}(0, x) \leqslant \frac{C}{s^{d / 2}} \mathrm{e}^{-c|x|^{2} / s}+C s^{-d}, \quad s \in \mathbb{N}, x \in \mathbb{Z}^{d} .
$$

This gives, for any $y \in B_{R \alpha_{n}}$,

$$
\begin{aligned}
& \sum_{m \in \mathbb{Z}^{d} \backslash\{0\}} \sum_{s \in \mathbb{N}_{0}: s \geqslant \gamma \alpha_{n}|m|} \mathrm{e}^{-s \alpha_{n}^{-2}} p_{s}\left(0, y+2 m R \alpha_{n}\right) \\
& \leqslant C \sum_{s \geqslant \gamma \alpha_{n}} \mathrm{e}^{-s \alpha_{n}^{-2}}\left[s^{-d / 2} \sum_{0<|m| \leqslant s /\left(\gamma \alpha_{n}\right)} \mathrm{e}^{-c|m|^{2} \alpha_{n}^{2} / s}+\left(\frac{s}{\alpha_{n}}\right)^{d} s^{-d}\right],
\end{aligned}
$$

where we interchanged the sums on $s$ and $m$, and we also used that $\left|y+2 m R \alpha_{n}\right| \geqslant|m| \alpha_{n}$ for $m \in \mathbb{Z}^{d} \backslash\{0\}$. Using the substitution $w=|m| \alpha_{n} / \sqrt{s}$, the sum on $m$ is estimated by

$$
\sum_{0<|m| \leqslant s /\left(\gamma \alpha_{n}\right)} \mathrm{e}^{-c|m|^{2} \alpha_{n}^{2} / s} \leqslant C\left(\frac{s}{\alpha_{n}^{2}}\right)^{d / 2} \int_{\alpha_{n} / \sqrt{s}}^{\sqrt{s} / \gamma} \mathrm{d} w w^{d-1} \mathrm{e}^{-c w^{2}} \leqslant C\left(\frac{s}{\alpha_{n}^{2}}\right)^{d / 2} .
$$

Since $\sum_{s \in \mathbb{N}_{0}} \mathrm{e}^{-s \alpha_{n}^{-2}} \leqslant C \alpha_{n}^{2}$, this implies that

$$
\sum_{m \in \mathbb{Z}^{d} \backslash\{0\}} \sum_{s \in \mathbb{N}_{0}: s \geqslant \gamma \alpha_{n}|m|} \mathrm{e}^{-s \alpha_{n}^{-2}} p_{s}\left(0, y+2 m R \alpha_{n}\right) \leqslant C \alpha_{n}^{2-d} .
$$

Use (3.21) and (3.23) in (3.19) to conclude.

The next step is a preparation for the estimate of the last term in (3.13).

Step 5. Let $Y$ be a random variable that satisfies

$$
\limsup _{r \rightarrow \infty} r^{-q} \log \mathbb{P}(Y>r)<0
$$

for some $q>1$.

(i) Fix $L>0$. Then there is $C_{M, L}>0$ such that $\lim _{M \rightarrow \infty} C_{M, L}=0$ such that, for every $n \in \mathbb{N}$ and $M>0$ and $b_{n} \geqslant 1$,

$$
\mathbb{E}\left[\left(Y-M b_{n}\right)_{+}^{L b_{n}^{q}}\right]^{1 /\left(L b_{n}^{q}\right)} \leqslant b_{n} C_{M, L} .
$$

(ii) There is a constant $C>0$ such that, for any $\mu \in \mathbb{N}$,

$$
\mathbb{E}\left[Y_{+}^{\mu}\right] \leqslant \mu^{\frac{1}{q} \mu} C^{\mu} .
$$

Proof. From our assumption on $Y$, we know that there are $C, D>0$ and $q>1$ such that $\mathbb{P}(Y>s) \leqslant C \mathrm{e}^{-D s^{q}}$ for all $s>0$.

Proof of (i). We write $L$ instead of $L b_{n}^{q}$ and have

$$
b_{n}^{-L} \mathbb{E}\left[\left(Y-M b_{n}\right)_{+}^{L}\right]=b_{n}^{-L} \int_{0}^{\infty} \mathbb{P}\left(\left(Y-M b_{n}\right)^{L}>t\right) \mathrm{d} t=L \int_{0}^{\infty} s^{L-1} \mathbb{P}\left(Y>(s+M) b_{n}\right) \mathrm{d} s .
$$


Now use the above estimate $\mathbb{P}\left(Y>(s+M) b_{n}\right) \leqslant C \exp \left\{-D(s+M)^{q} b_{n}^{q}\right\}$ for all $s>0$. Furthermore, use that $(s+M)^{q} \geqslant s^{q}+M^{q}$. This gives

$$
\mathbb{E}\left[\left(Y-M b_{n}\right)_{+}^{L}\right] \leqslant b_{n}^{L} L C \mathrm{e}^{-D M^{q} b_{n}^{q}} \int_{0}^{\infty} s^{L-1} \mathrm{e}^{-D\left(s b_{n}\right)^{q}} \mathrm{~d} s=L C \mathrm{e}^{-D M^{q} b_{n}^{q}} \int_{0}^{\infty} s^{L-1} \mathrm{e}^{-D s^{q}} \mathrm{~d} s .
$$

The change of variables $t=D s^{q}$ turns this into

$$
\mathbb{E}\left[\left(Y-M b_{n}\right)_{+}^{L}\right] \leqslant L C \mathrm{e}^{-D M^{q} b_{n}^{q}} D^{-L / q} q \Gamma(L / q),
$$

where $\Gamma$ denotes the Gamma-function. Note that $\Gamma(x) \leqslant\left(C_{1} x\right)^{x}$ for some $C_{1}>0$ and all $x \geqslant 1$. Now we replace $L$ by $L b_{n}^{q}$ and take the $\left(L b_{n}^{q}\right)$-th root to obtain

$$
\mathbb{E}\left[\left(Y-M b_{n}\right)_{+}^{L b_{n}^{q}}\right]^{1 /\left(L b_{n}^{q}\right)} \leqslant \widetilde{C}\left(L b_{n}^{q}\right)^{1 /\left(L b_{n}^{q}\right)} L^{1 / q} \mathrm{e}^{-M^{q} D / L} b_{n},
$$

where $\widetilde{C}$ does not depend on $L$ nor on $M$ or $n$. Since $b_{n} \geqslant 1$, the assertion is proved.

Proof of (ii). From (3.29) with $M=0$ and $L=\mu$, we have $\mathbb{E}\left[Y_{+}^{\mu}\right] \leqslant \mu C D^{-\mu / q} \Gamma(\mu / q)$. Recalling that $\Gamma(x) \leqslant$ $\left(C_{1} x\right)^{x}$ for some $C_{1}>0$ and all $x \geqslant 1$, we arrive at the assertion.

Step 6. Conclusion of the proof.

Proof. Fix $R>0$ and let $B=B_{R \alpha_{n}}$ be the centered box in $\mathbb{Z}^{d}$ with radius $R \alpha_{n}$. Note that in both cases (V) and (L), (3.24) is satisfied with $q>\frac{d}{2}$. Let $p$ be defined by $1=\frac{1}{p}+\frac{1}{q}$. Then, in both cases, $p \in\left(1, \frac{d}{d-2}\right)$ if $d \geqslant 3$ and $p>1$ if $d=2$. Put $k=n \alpha_{n}^{-2}$. Recall that $\alpha_{n}^{d+2}=n b_{n}^{-q}$. Recall that it suffices to prove (3.12). In the following, we shall use $C$ to denote a generic positive constant which depends on $R, q$ and $D$ only and may change its value from line to line.

Use Steps 2-3 for the scenery $Y$ replaced by $Y^{\left(>M b_{n}\right)}$ and $R$ replaced by $R \alpha_{n}$ and with $\lambda=\alpha_{n}^{-2}$ to obtain

$$
\mathbb{E}\left[\left\langle\ell_{n}, Y^{\left(>M b_{n}\right)}\right\rangle^{k}\right] \leqslant \mathrm{e}^{k} \sum_{\sigma \in \mathfrak{S}_{k}} \sum_{z_{1}, \ldots, z_{k} \in B} \prod_{i=1}^{k} G_{\alpha_{n}^{-2}}^{\left(R \alpha_{n}\right)}\left(z_{\sigma(i-1)}, z_{\sigma(i)}\right) \prod_{x \in B} \mathbb{E}\left[\left(Y(0)-M b_{n}\right)_{+}^{\mu_{x}}\right],
$$

where we abbreviated $\mu_{x}=\#\left\{i: z_{i}=x\right\}$. Let us estimate the last term. We fix a parameter $L>0$ and split the product on $x \in B$ into the subproducts on $B_{(L)}=\left\{x \in B: \mu_{x} \leqslant L b_{n}^{q}\right\}$ and $B_{(L)}^{\mathrm{c}}=B \backslash B_{(L)}$. We estimate, with the help of Step 5,

$$
\begin{aligned}
\prod_{x \in B} \mathbb{E}\left[\left(Y(0)-M b_{n}\right)_{+}^{\mu_{x}}\right] & \leqslant \prod_{x \in B_{(L)}} \mathbb{E}\left[\left(Y(0)-M b_{n}\right)_{+}^{L b_{n}^{q}}\right]^{\mu_{x} /\left(L b_{n}^{q}\right)} \prod_{x \in B_{(L)}^{\mathfrak{c}}} \mathbb{E}\left[Y(0)_{+}^{\mu_{x}}\right] \\
& \leqslant \prod_{x \in B_{(L)}}\left(C_{M, L} b_{n}\right)^{\mu_{x}} \prod_{x \in B_{(L)}^{\mathbf{c}}}\left(C \mu_{x}^{1 / q}\right)^{\mu_{x}} .
\end{aligned}
$$

Let us abbreviate the term on the right-hand side by $K(\mu)$ where $\mu=\left(\mu_{x}\right)_{x \in B}$. Now we pick numbers $p^{\prime}>p, q^{\prime}>1$ such that $\frac{1}{p^{\prime}}+\frac{1}{q^{\prime}}=1$ and, if $d \geqslant 3, p^{\prime}<\frac{d}{d-2}$, and use Hölder's inequality in (3.30) to obtain

$$
\mathbb{E}\left[\left\langle\ell_{n}, Y^{\left(>M b_{n}\right)}\right\rangle^{k}\right] \leqslant \mathrm{e}^{k} \sum_{\sigma \in \mathfrak{S}_{k}}\left(\sum_{z_{1}, \ldots, z_{k} \in B} \prod_{i=1}^{k} G_{\alpha_{n}^{-2}}^{\left(R \alpha_{n}\right)}\left(z_{\sigma(i-1)}, z_{\sigma(i)}\right)^{p^{\prime}}\right)^{1 / p^{\prime}}\left(\sum_{z_{1}, \ldots, z_{k} \in B} K(\mu)^{q^{\prime}}\right)^{1 / q^{\prime}} .
$$

Using (3.18) in Step 4, the term in the first brackets may be estimated by

$$
\left(\sum_{z_{1}, \ldots, z_{k} \in B} \prod_{i=1}^{k} G_{\alpha_{n}^{-2}}^{\left(R \alpha_{n}\right)}\left(z_{\sigma(i-1)}, z_{\sigma(i)}\right)^{p^{\prime}}\right)^{1 / p^{\prime}} \leqslant C^{k} \alpha_{n}^{2 k} \alpha_{n}^{-\frac{1}{q^{\prime}} d k} .
$$

Now we estimate the last term in (3.32). By $A_{k}$ we denote the set of maps $\mu: B \rightarrow \mathbb{N}_{0}$ such that $\sum_{x \in B} \mu_{x}=k$. Observe that, for any $\mu \in A_{k}$, we have

$$
\#\left\{\left(z_{1}, \ldots, z_{k}\right) \in B^{k}: \mu_{x}=\#\left\{i: z_{i}=x\right\} \forall x \in B\right\}=\frac{k !}{\prod_{x \in B} \mu_{x} !} .
$$


Hence,

$$
\sum_{z_{1}, \ldots, z_{k} \in B} K(\mu)^{q^{\prime}} \leqslant C^{k} k ! \sum_{\mu \in A_{k}} \prod_{x \in B_{(L)}} C_{M, L}^{q^{\prime} \mu_{x}} \prod_{x \in B_{(L)}}\left(\frac{b_{n}^{q^{\prime}}}{\mu_{x}}\right)^{\mu_{x}} \prod_{x \in B_{(L)}^{\mathrm{c}}} \mu_{x}^{-\left(1-q^{\prime} / q\right) \mu_{x}} .
$$

Since $q^{\prime}<q$, we have that $r \equiv 1-\frac{q^{\prime}}{q}$ is positive. According to the definition of $B_{(L)}$, the last term in (3.34) can be estimated by

$$
\prod_{x \in B_{(L)}^{\mathrm{c}}} \mu_{x}^{-\left(1-q^{\prime} / q\right) \mu_{x}} \leqslant \prod_{x \in B_{(L)}^{\mathrm{c}}}\left(L^{-r} b_{n}^{\left(q^{\prime}-q\right)}\right)^{\mu_{x}} .
$$

The penultimate term in (3.34) can be estimated as

$$
\prod_{x \in B_{(L)}}\left(\frac{b_{n}^{q^{\prime}}}{\mu_{x}}\right)^{\mu_{x}} \leqslant C^{k} \prod_{x \in B_{(L)}} b_{n}^{\left(q^{\prime}-q\right) \mu_{x}},
$$

since we have, using also Jensen's inequality for the logarithm,

$$
\begin{aligned}
\prod_{x \in B_{(L)}}\left(\frac{b_{n}^{q^{\prime}}}{\mu_{x}}\right)^{\mu_{x}} & =\exp \left\{\left(\sum_{y \in B_{(L)}} \mu_{y}\right) \sum_{x \in B_{(L)}} \frac{\mu_{x}}{\sum_{y \in B_{(L)}} \mu_{y}} \log \frac{b_{n}^{q^{\prime}}}{\mu_{x}}\right\} \\
& \leqslant \exp \left\{\left(\sum_{y \in B_{(L)}} \mu_{y}\right) \log \sum_{x \in B_{(L)}} \frac{b_{n}^{q^{\prime}}}{\sum_{y \in B_{(L)}} \mu_{y}}\right\} \\
& =\prod_{x \in B_{(L)}}\left(\frac{b_{n}^{q^{\prime}} \# B_{(L)}}{\sum_{y \in B_{(L)}} \mu_{y}}\right)^{\mu_{x}} .
\end{aligned}
$$

Now use that $\# B_{(L)} \leqslant \# B \leqslant C \alpha_{n}^{d}=C k b_{n}^{-q}$ and observe that there is a constant $C>0$ such that $\left(\frac{k}{l}\right)^{l} \leqslant C^{k}$, for any $l \in\{1, \ldots, k\}$, since the map $y \mapsto y \log y$ is bounded on $(0,1]$. Using (3.35) and (3.36) in (3.34), we obtain, for some constant $C_{M}>0$, satisfying $\lim _{M \rightarrow \infty} C_{M}=0$,

$$
\begin{aligned}
\sum_{z_{1}, \ldots, z_{k} \in B} K(\mu)^{q^{\prime}} & \leqslant C^{k} k ! b_{n}^{\left(q^{\prime}-q\right) k} \sum_{\mu \in A_{k}} \prod_{x \in B_{(L)}} C_{M, L}^{q^{\prime} \mu_{x}} \prod_{x \in B_{(L)}^{\mathrm{c}}} L^{-r \mu_{x}} \\
& \leqslant C^{k} k ! b_{n}^{\left(q^{\prime}-q\right) k} \# A_{k}\left(\max \left\{C_{M, L}^{q^{\prime}}, L^{-r}\right\}\right)^{k} \leqslant C_{M}^{q^{\prime} k} k ! b_{n}^{\left(q^{\prime}-q\right) k},
\end{aligned}
$$

where we choose $L$ in dependence on $M$ such that $\lim _{M \rightarrow \infty} \max \left\{C_{M, L}^{q^{\prime}}, L^{-r}\right\}=0$, and we estimated \# $A_{k}=\left(\begin{array}{c}k+|B| \\ |B|\end{array}\right) \leqslant$ $\mathrm{e}^{\mathrm{o}(k)}$ (recall that $\left.k=n \alpha_{n}^{2}\right)$.

Using (3.37) and (3.33) in (3.32), we arrive at

$$
\mathbb{E}\left[\left\langle\ell_{n}, Y^{\left(>M b_{n}\right)}\right\rangle^{k}\right] \leqslant C_{M}^{k} k ! \alpha_{n}^{2} k \alpha_{n}^{-\frac{1}{q^{\prime}} d k}\left(k ! b_{n}^{\left(q^{\prime}-q\right) k}\right)^{1 / q^{\prime}} .
$$

Now recall that $b_{n}^{q} \alpha_{n}^{d}=k=n \alpha_{n}^{-2}$ and use Stirling's formula to see that the right-hand side of this estimate is bounded from above by $C_{M}^{k}\left(n b_{n}\right)^{k}$ for some $C_{M} \rightarrow 0$ as $M \rightarrow \infty$. This ends the proof of Proposition 3.4.

\subsection{Smoothing the scenery}

In this section we provide the smoothing argument for the field. This will be an adaptation of results of [1, Section 3]. Fix some smooth, rotationally invariant, and $L^{1}$-normalized function $\kappa: \mathbb{R}^{d} \rightarrow[0, \infty)$ with $\operatorname{supp}(\kappa) \subset Q_{1}$, and put $\kappa_{\delta}(\cdot)=\delta^{-d} \kappa(\cdot / \delta)$ for some small $\delta>0$. The convolution of two functions $f, g: \mathbb{R}^{d} \rightarrow \mathbb{R}$ is denoted by $f * g$. Assume any of the cases (V) and (L) and choose $\left(b_{n}\right)_{n}$ and $\left(\alpha_{n}\right)_{n}$ according to (1.27). We consider the rescaled and cut-down field $\bar{Y}_{n}^{(\leqslant M)}: \mathbb{R}^{d} \rightarrow[-M, M]$; see (3.10). Recall the scaled and normalized local times $L_{n}$ from (1.19). By $\mathcal{B}_{M}\left(\mathbb{R}^{d}\right)$ we denote the set of all measurable functions $\mathbb{R}^{d} \rightarrow[-M, M]$. 
Lemma 3.5 (Scenery smoothing). Fix $M>0$. Then, for any $\varepsilon>0$,

$$
\lim _{\delta \downarrow 0} \limsup _{n \rightarrow \infty} \frac{\alpha_{n}^{2}}{n} \log \sup _{f \in \mathcal{B}_{M}\left(\mathbb{R}^{d}\right)} \mathbb{P}\left(\left|\left\langle L_{n},\left[f-f * \kappa_{\delta}\right]\right\rangle\right|>\varepsilon\right)=-\infty .
$$

In particular,

$$
\lim _{\delta \downarrow 0} \limsup _{n \rightarrow \infty} \frac{\alpha_{n}^{2}}{n} \log \mathbb{P}\left(\left|\left\langle L_{n},\left[\bar{Y}_{n}^{(\leqslant M)}-\bar{Y}_{n}^{(\leqslant M)} * \kappa_{\delta}\right]\right\rangle\right|>\varepsilon\right)=-\infty .
$$

Proof. Certainly, it suffices to prove (3.39) for $M=1$. We adapt the proof of [1, Lemma 3.1], which is the same statement for $M=1$ and Brownian motion instead of random walk in Brownian scaling. We shall write $\mathcal{B}$ instead of $\mathcal{B}_{1}\left(\mathbb{R}^{d}\right)$.

Since all exponential moments of the steps are assumed finite, we have

$$
\lim _{R \rightarrow \infty} \limsup _{n \rightarrow \infty} \frac{\alpha_{n}^{2}}{n} \log \mathbb{P}\left(\operatorname{supp}\left(\ell_{n}\right) \not \subset B_{R_{n}}\right)=-\infty,
$$

where $R_{n}=R n \alpha_{n}^{-1}$. Hence, it suffices to show, for every $R>0$,

$$
\lim _{\delta \downarrow 0} \limsup \frac{\alpha_{n}^{2}}{n} \log \sup _{f \in \mathcal{B}} \mathbb{P}\left(\left|\left\langle L_{n}, f-f * \kappa_{\delta}\right\rangle\right|>\varepsilon, \operatorname{supp}\left(\ell_{n}\right) \subset B_{R_{n}}\right)=-\infty .
$$

We prove this only without absolute value signs, since the complementary inequality is proved in the same way. Fix $f \in \mathcal{B}$. Chebyshev's inequality yields, for any $a>0$,

$$
\begin{aligned}
& \mathbb{P}\left(\left\langle L_{n}, f-f * \kappa_{\delta}\right\rangle>\varepsilon, \operatorname{supp}\left(\ell_{n}\right) \subset B_{R_{n}}\right) \\
& \quad \leqslant \mathbb{E}\left[\exp \left\{a \frac{n}{\alpha_{n}^{2}}\left\langle L_{n}, f-f * \kappa_{\delta}\right\rangle\right\} \mathbb{1}\left\{\operatorname{supp}\left(\ell_{n}\right) \subset B_{R_{n}}\right\}\right] \mathrm{e}^{-a \varepsilon n \alpha_{n}^{-2}} .
\end{aligned}
$$

Introduce a discrete version $\varphi_{n}: \mathbb{Z}^{d} \rightarrow \mathbb{R}$ of $f-f * \kappa_{\delta}$ by

$$
\varphi_{n}(z)=\alpha_{n}^{d} \int_{z \alpha_{n}^{-1}+\left[0, \alpha_{n}^{-1}\right)^{d}}\left[f-f * \kappa_{\delta}\right](x) \mathrm{d} x, \quad z \in \mathbb{Z}^{d} .
$$

Note that

$$
\begin{aligned}
& \frac{n}{\alpha_{n}^{2}}\left\langle L_{n}, f-f * \kappa_{\delta}\right\rangle=\alpha_{n}^{d-2} \int\left[f-f * \kappa_{\delta}\right](x) \ell_{n}\left(\left\lfloor x \alpha_{n}\right\rfloor\right) \mathrm{d} x=\alpha_{n}^{-2} \sum_{z \in \mathbb{Z}^{d}} \ell_{n}(z) \varphi_{n}(z) \\
& \quad=\alpha_{n}^{-2} \sum_{k=0}^{n} \varphi_{n}\left(S_{k}\right) .
\end{aligned}
$$

We first express the expectation on the right side of (3.42) in terms of an expansion with respect to an appropriate orthonormal system of eigenvalues and eigenfunctions in $\mathbb{R}^{B_{R_{n}}}$. We write $\mathbb{E}_{z}$ for expectation with respect to the random walk when started at $z \in \mathbb{Z}^{d}$, in particular $\mathbb{E}=\mathbb{E}_{0}$. By (3.44), for any $z, \tilde{z} \in B_{R_{n}}$,

$$
\mathbb{E}_{z}\left[\exp \left\{a \frac{n}{\alpha_{n}^{2}}\left\langle L_{n}, f-f * \kappa_{\delta}\right\rangle\right\} \mathbb{1}\left\{\operatorname{supp}\left(\ell_{n}\right) \subset B_{R_{n}}\right\} \mathbb{1}\left\{S_{n}=\tilde{z}\right\}\right]=\mathrm{e}^{\frac{a}{2} \alpha_{n}^{-2}\left(\varphi_{n}(z)+\varphi_{n}(\tilde{z})\right)} A^{n}(z, \tilde{z}),
$$

where $A^{n}$ is the $n$-th power of the symmetric matrix $A$ having components

$$
A(z, \tilde{z})=\mathrm{e}^{\frac{a}{2} \alpha_{n}^{-2} \varphi_{n}(z)} p(z, \tilde{z}) \mathrm{e}^{\frac{a}{2} \alpha_{n}^{-2} \varphi_{n}(\tilde{z})}, \quad z, \tilde{z} \in B_{R_{n}} .
$$

Using an expansion in terms of the eigenvalues $\lambda_{k}(n), k \in\left\{1, \ldots,\left|B_{R_{n}}\right|\right\}$, of $A$ and an orthonormal basis of $\mathbb{R}^{B_{R_{n}}}$ consisting of corresponding eigenfunctions $v_{k, n}$ we obtain, for any $z, \tilde{z} \in B_{R_{n}}$,

$$
A^{n}(z, \tilde{z})=\sum_{k=1}^{\left|B_{R_{n}}\right|} \lambda_{k}(n)^{n} v_{k, n}(z) v_{k, n}(\tilde{z}) .
$$


We assume that the eigenvalues $\lambda_{k}(n)$ are in decreasing order, and the principal eigenvector $v_{1, n}$ is positive in $B_{R_{n}}$.

Now we use this for the expectation on the right side of (3.42), which is equal to the sum over $\tilde{z} \in B_{R_{n}}$ of the left side of (3.45) at $z=0$. We obtain an upper bound by summing the right-hand side of (3.47) over $z, \tilde{z} \in B_{R_{n}}$. Continuing the upper bound with the help of Parseval's identity gives

$$
\begin{aligned}
& \mathbb{E}\left[\exp \left\{a \frac{n}{\alpha_{n}^{2}}\left\langle L_{n}, f-f * \kappa_{\delta}\right\rangle\right\} \mathbb{1}\left\{\operatorname{supp}\left(\ell_{n}\right) \subset B_{R_{n}}\right\}\right] \\
& \leqslant(1+\mathrm{o}(1)) \sum_{k=1}^{\left|B_{R_{n}}\right|} \lambda_{k}(n)^{n} \sum_{z, \tilde{z} \in B_{R_{n}}} v_{k, n}(z) v_{k, n}(\tilde{z}) \leqslant(1+\mathrm{o}(1)) \lambda_{1}(n)^{n} \sum_{k=1}^{\left|B_{R_{n}}\right|}\left\langle v_{k, n}, \mathbb{1}\right\rangle^{2} \\
& \quad \leqslant(1+\mathrm{o}(1)) \lambda_{1}(n)^{n}\left|B_{R_{n}}\right|,
\end{aligned}
$$

where we denote by $\langle\cdot, \cdot\rangle$ and $\|\cdot\|_{2}$ the inner product and Euclidean norm on $\mathbb{R}^{B_{R_{n}}}$. Recall that $R_{n}=R n \alpha_{n}^{-1}$. Our assumptions on $\left(\alpha_{n}\right)_{n}$ imply that $\left|B_{R_{n}}\right|=\mathrm{e}^{\mathrm{o}\left(n \alpha_{n}^{-2}\right)}$ as $n \rightarrow \infty$. Hence, as $n \rightarrow \infty$,

$$
\frac{\alpha_{n}^{2}}{n} \log \mathbb{E}\left[\exp \left\{a \frac{n}{\alpha_{n}^{2}}\left\langle L_{n}, f-f * \kappa_{\delta}\right\rangle\right\} \mathbb{1}\left\{\operatorname{supp}\left(\ell_{n}\right) \subset B_{R_{n}}\right\}\right] \leqslant \mathrm{o}(1)+\alpha_{n}^{2}\left[\lambda_{1}(n)-1\right] .
$$

Recall the Rayleigh-Ritz principle, $\lambda_{1}(n)=\max _{\|g\| \leqslant 1}\langle A g, g\rangle$, where the maximum runs over all $\ell^{2}$-normalized vectors $g: \mathbb{Z}^{d} \rightarrow(0, \infty)$ with support in $B_{R_{n}}$. Recall that $\left|\varphi_{n}\right| \leqslant 2$. Then, as $n \rightarrow \infty$, we have, for any $\ell^{2}$-normalized vector $g$,

$$
\begin{aligned}
\alpha_{n}^{2}[\langle A g, g\rangle-1] & =\alpha_{n}^{2}\left(\sum_{z, \tilde{z}}\left(\mathrm{e}^{\frac{a}{2} \alpha_{n}^{-2}\left[\varphi_{n}(z)+\varphi_{n}(\tilde{z})\right]}-1\right) p(z, \tilde{z}) g(z) g(\tilde{z})+\sum_{z, \tilde{z}}\left(p(z, \tilde{z})-\delta_{z, \tilde{z}}\right) g(z) g(\tilde{z})\right) \\
& =a\left\langle\varphi_{n}, g^{2}\right\rangle+a\left\langle\varphi_{n}, g(p g-g)\right\rangle+\mathcal{O}\left(\alpha_{n}^{-2}\right)-\alpha_{n}^{2} \mathcal{I}^{(\mathrm{d})}\left(g^{2}\right),
\end{aligned}
$$

where we recall that the walk is assumed symmetric, and we introduced its Dirichlet form,

$$
\mathcal{I}^{(\mathrm{d})}\left(g^{2}\right)=\frac{1}{2} \sum_{z, \tilde{z} \in \mathbb{Z}^{d}} p(z, \tilde{z})(g(z)-g(\tilde{z}))^{2}, \quad g \in \ell_{2}\left(\mathbb{Z}^{d}\right),
$$

and we wrote $\operatorname{pg}(z)=\sum_{\tilde{z}} p(z, \tilde{z}) g(\tilde{z})$.

The second term on the right-hand side of (3.50) is estimated as follows, using that $\left|\varphi_{n}\right| \leqslant 2$.

$$
\begin{aligned}
\left\langle\varphi_{n}, g(p g-g)\right\rangle & =\frac{1}{2} \sum_{z, \tilde{z}} \varphi_{n}(z) p(z, \tilde{z})\left[-(g(z)-g(\tilde{z}))^{2}+(g(\tilde{z})-g(z))(g(z)+g(\tilde{z}))\right] \\
& \leqslant 2 \mathcal{I}^{(\mathrm{d})}\left(g^{2}\right)+\sqrt{2 \mathcal{I}^{(\mathrm{d})}\left(g^{2}\right)} \sqrt{\frac{1}{2} \sum_{z, \tilde{z}}\left|\varphi_{n}(z)\right| p(z, \tilde{z})(g(z)+g(\tilde{z}))^{2}} \\
& \leqslant 2 \mathcal{I}^{(\mathrm{d})}\left(g^{2}\right)+\frac{8}{\varepsilon} \mathcal{I}^{(\mathrm{d})}\left(g^{2}\right)+\frac{\varepsilon}{4},
\end{aligned}
$$

where we used the inequality $\sqrt{2 a b} \leqslant 8 a / \varepsilon+\varepsilon b / 16$ for $a, b, \varepsilon>0$ in the last step.

The first term on the right-hand side of (3.50) is estimated as follows. We introduce $g_{n}(x)=g\left(\left\lfloor x \alpha_{n}\right\rfloor\right)$.

$$
\begin{aligned}
\left\langle\varphi_{n}, g^{2}\right\rangle & =\alpha_{n}^{d} \int \mathrm{d} x f(x)\left(g_{n}^{2}(x)-\int \mathrm{d} y \kappa_{\delta}(y) g_{n}^{2}(x+y)\right) \leqslant \alpha_{n}^{d} \int \mathrm{d} x \int \mathrm{d} y \kappa_{\delta}(y)\left|g_{n}^{2}(x)-g_{n}^{2}(x+y)\right| \\
& \leqslant \alpha_{n}^{d} \int \mathrm{d} x \sqrt{\int \mathrm{d} y \kappa_{\delta}(y)\left(g_{n}(x)-g_{n}(x+y)\right)^{2}} \sqrt{\int \mathrm{d} y \kappa_{\delta}(y)\left(g_{n}(x)+g_{n}(x+y)\right)^{2}} \\
& \leqslant \frac{4}{\varepsilon} \alpha_{n}^{d} \int \mathrm{d} x \int \mathrm{d} y \kappa_{\delta}(y)\left(g_{n}(x)-g_{n}(x+y)\right)^{2}+\frac{\varepsilon}{8} \alpha_{n}^{d} \int \mathrm{d} x\left(g_{n}^{2}(x)+\int \mathrm{d} y \kappa_{\delta}(y) g_{n}^{2}(x+y)\right) \\
& \leqslant \frac{4}{\varepsilon} \alpha_{n}^{d} \int \mathrm{d} x \int \mathrm{d} y \kappa_{\delta}(y)\left(g_{n}(x)-g_{n}(x+y)\right)^{2}+\frac{\varepsilon}{4},
\end{aligned}
$$


where we used that $|f| \leqslant 1$ in the second step, Hölder's inequality in the third, and the inequality $\sqrt{2 a b} \leqslant 4 a / \varepsilon+\varepsilon b / 8$ in the fourth step. Now pick some almost everywhere differentiable function $\psi_{n}: \mathbb{R}^{d} \rightarrow \mathbb{R}$ such that $\psi_{n}\left(z / \alpha_{n}\right)=$ $\alpha_{n}^{d / 2} g(z)$ for any $z \in \mathbb{Z}^{d}$, then a Taylor expansion gives that

$$
\begin{aligned}
\alpha_{n}^{d} \int \mathrm{d} x \int \mathrm{d} y \kappa_{\delta}(y)\left(g_{n}(x)-g_{n}(x+y)\right)^{2} & =\alpha_{n}^{-d} \sum_{z, \tilde{z}}\left(\psi_{n}\left(\frac{z}{\alpha_{n}}\right)-\psi_{n}\left(\frac{z+\tilde{z}}{\alpha_{n}}\right)\right)^{2} \int_{\tilde{z} / \alpha_{n}+\left[0,1 / \alpha_{n}\right]^{d}} \mathrm{~d} y \kappa_{\delta}(y) \\
& =\alpha_{n}^{-d} \sum_{z, \tilde{z}}\left(\int_{0}^{1} \mathrm{~d} t \frac{\tilde{z}}{\alpha_{n}} \cdot \nabla \psi_{n}\left(\frac{z+t \tilde{z}}{\alpha_{n}}\right)\right)^{2} \int_{\tilde{z} / \alpha_{n}+\left[0,1 / \alpha_{n}\right]^{d}} \mathrm{~d} y \kappa_{\delta}(y) \\
& \leqslant \alpha_{n}^{-d} \sum_{\tilde{z}} \int_{\tilde{z} / \alpha_{n}+\left[0,1 / \alpha_{n}\right]^{d}} \mathrm{~d} y \kappa_{\delta}(y)\left|\frac{\tilde{z}}{\alpha_{n}}\right|_{0}^{2} \mathrm{~d} t \sum_{z}^{1} \mid \nabla \psi_{n}\left(\frac{z+t \tilde{z}}{\alpha_{n}}\right)^{2} \\
& \leqslant C \delta^{2}\left\|\nabla \psi_{n}\right\|_{2}^{2} \leqslant C \delta^{2}\left\|\Gamma^{1 / 2} \nabla \psi_{n}\right\|_{2}^{2},
\end{aligned}
$$

where we remark that $\int \mathrm{d} y \kappa_{\delta}(y)|y|^{2} \leqslant C \delta^{2}$ for some $C>0$. Now we specialize the choice of $\psi_{n}$ to

$$
\psi_{n}(x)=\alpha_{n}^{d / 2}\left[g_{n}(x)+\sum_{i=1}^{d}\left(\alpha_{n} x_{i}-\left\lfloor\alpha_{n} x_{i}\right\rfloor\right)\left(g\left(\left\lfloor\alpha_{n} x\right\rfloor+\mathrm{e}_{i}\right)-g\left(\left\lfloor\alpha_{n} x\right\rfloor\right)\right)\right],
$$

where $\mathrm{e}_{i}$ denotes the $i$-th unit vector. Then $\psi_{n}$ is the linear interpolation of the rescaling of $g$, and $\partial_{i} \psi_{n}(x)=$ $\alpha_{n}^{d / 2+1}\left(g\left(\left\lfloor\alpha_{n} x\right\rfloor+\mathrm{e}_{i}\right)-g\left(\left\lfloor\alpha_{n} x\right\rfloor\right)\right)$. Similarly to (3.54), one derives

$$
\begin{aligned}
\alpha_{n}^{2} \mathcal{I}^{(\mathrm{d})}\left(g^{2}\right) & =\int_{0}^{1} \mathrm{~d} t \int_{0}^{1} \mathrm{~d} s \sum_{z \in \mathbb{Z}^{d}} p(0, z) \sum_{i, j=1}^{d} z_{i} z_{j} \int \mathrm{d} x \partial_{i} \psi_{n}\left(\frac{\left\lfloor\alpha_{n} x\right\rfloor+t z}{\alpha_{n}}\right) \partial_{j} \psi_{n}\left(\frac{\left\lfloor\alpha_{n} x\right\rfloor+s z}{\alpha_{n}}\right) \\
& =\int_{0}^{1} \mathrm{~d} t \int_{0}^{1} \mathrm{~d} s \sum_{z} p(0, z) \sum_{i, j=1}^{d} z_{i} z_{j} \int \mathrm{d} x \partial_{i} \psi_{n}(x) \partial_{j} \psi_{n}(x) \\
& =\left\|\Gamma^{1 / 2} \nabla \psi_{n}\right\|_{2}^{2} .
\end{aligned}
$$

Now use (3.55) in (3.54) and this in (3.53), and substitute (3.53) and (3.52) in (3.50) to obtain, for any $a>0$, for $n$ sufficiently large and all $\ell^{2}$-normalized $g \in \ell^{2}\left(\mathbb{Z}^{d}\right)$ with support in $B_{R_{n}}$,

$$
\alpha_{n}^{2}[\langle A g, g\rangle-1] \leqslant \frac{1}{2} a \varepsilon-\alpha_{n}^{2} \mathcal{I}^{(\mathrm{d})}\left(g^{2}\right)\left(1-C \frac{\delta^{2} a}{\varepsilon}\right),
$$

for some $C>0$ which does not depend on $n, g, \varepsilon$ or on $a$. Now we choose $a=\varepsilon /\left(2 C \delta^{2}\right)$ and obtain $\alpha_{n}^{2}[\langle A g, g\rangle-1] \leqslant$ $\frac{1}{2} a \varepsilon$. Taking the supremum over all $g$ 's considered, we obtain that $\alpha_{n}^{2}\left[\lambda_{1}(n)-1\right] \leqslant \frac{1}{2} a \varepsilon$. Using this in (3.49) and (3.49) in (3.42), we obtain that

$$
\text { 1.h.s. of }(3.40) \leqslant \limsup _{\delta \downarrow 0}-\frac{1}{2} a \varepsilon=-\lim _{\delta \downarrow 0} \frac{\varepsilon^{2}}{4 C \delta^{2}}=-\infty,
$$

and the proof is finished.

\subsection{Various approximations}

In the proofs of Theorems 1.1 and 1.3 we shall need a couple of approximations to the variational formulas in (1.12) and (1.10). In particular, we need to show that they may be approximated by finite-space approximations and by smoothed versions of the functions involved in the variational formula.

As in Section 3.4, by $\kappa=\kappa_{1}: \mathbb{R}^{d} \rightarrow[0, \infty)$ we denote a smooth, rotationally invariant $L^{1}$-normalized function, and we put $\kappa_{\delta}(x)=\delta^{-d} \kappa_{1}\left(x \delta^{-1}\right)$ for $\delta>0$. Hence, $\kappa_{\delta}$ is a smooth approximation of the Dirac measure at zero. 
Lemma 3.6 (Approximations of $K_{H}$ ). For any $u>0$,

$$
\underset{\delta \downarrow 0}{\limsup \limsup } K_{R \rightarrow \infty}^{(0)}(u ; \delta, R) \leqslant K_{H}(u) \leqslant \liminf _{\delta \downarrow} \liminf _{R \rightarrow \infty} K_{H}^{(\text {per })}(u ; \delta, R),
$$

where

$$
\begin{gathered}
K_{H}^{(0)}(u ; \delta, R)=\inf \left\{\frac{1}{2}\left\|\Gamma^{1 / 2} \nabla \psi\right\|_{2}^{2}+\Phi_{H}\left(\psi^{2} * \kappa_{\delta}, u ; R\right): \psi \in H^{1}\left(\mathbb{R}^{d}\right), \operatorname{supp}(\psi) \subset Q_{R},\|\psi\|_{2}=1\right\}, \\
K_{H}^{(\text {per })}(u ; \delta, R)=\inf \left\{\frac{1}{2}\left\|\Gamma^{1 / 2} \nabla_{R} \psi\right\|_{2}^{2}+\Phi_{H}\left(\psi^{2} * \kappa_{\delta}, u ; R\right): \psi \in H^{1}\left(Q_{R}\right),\|\psi\|_{2}=1\right\},
\end{gathered}
$$

and $\Phi_{H}\left(\psi^{2}, u ; R\right)$ is defined in (3.9). In (3.58), $\nabla_{R}$ denotes the gradient on the torus $Q_{R}$, i.e., with periodic boundary condition.

Proof. Fix $\delta>0$. In the first step, we carry out the limit as $R \rightarrow \infty$ on both sides to obtain

$$
\limsup _{R \rightarrow \infty} K_{H}^{(0)}(u ; \delta, R) \leqslant K_{H}(u ; \delta) \leqslant \liminf _{R \rightarrow \infty} K_{H}^{(\text {per })}(u ; \delta, R),
$$

where $K_{H}(u ; \delta)$ is defined as $K_{H}(u)$ in (1.12) with $\Phi_{H}\left(\psi^{2}, u\right)$ replaced by $\Phi_{H}\left(\psi^{2} * \kappa_{\delta}, u\right)$. The proof of (3.59) follows standard patterns (see the proof of [1, Lemma 3.7], e.g.) and we do not carry this out here. Hence, the only thing left to do is to show that $\lim _{\delta \downarrow 0} K_{H}(u ; \delta)=K_{H}(u)$.

Using the convexity of $H$, it is easy to derive with the help of Jensen's inequality that, for any $\gamma>0$ and any $\psi$,

$$
\int H\left(\gamma \psi^{2} * \kappa_{\delta}(y)\right) \mathrm{d} y \leqslant \int H\left(\gamma \psi^{2}(y)\right) \mathrm{d} y .
$$

As a consequence, we have $\Phi_{H}\left(\psi^{2} * \kappa_{\delta}, u\right) \geqslant \Phi_{H}\left(\psi^{2}, u\right)$ and therefore $K_{H}(u ; \delta) \geqslant K_{H}(u)$ for any $\delta>0$.

We argue now that $\lim \sup _{\delta \downarrow 0} K_{H}(u ; \delta) \leqslant K_{H}(u)$. Indeed, fix some small $\varepsilon>0$ and pick some bounded approximative $\varepsilon$-minimizer for $K_{H}(u)$, i.e., a bounded function $\bar{\psi} \in H^{1}\left(\mathbb{R}^{d}\right)$ satisfying $\|\bar{\psi}\|_{2}=1$ and

$$
\frac{1}{2}\left\|\Gamma^{1 / 2} \nabla \bar{\psi}\right\|_{2}^{2}+\Phi_{H}\left(\bar{\psi}^{2}, u\right) \leqslant K_{H}(u)+\varepsilon
$$

Using the mean-value theorem and the fact that $\left\|\bar{\psi}^{2} * \kappa_{\delta}-\bar{\psi}^{2}\right\|_{1} \rightarrow 0$ as $\delta \downarrow 0$ (see [21, Theorem 2.16]), it is elementary to show that we have $\int H\left(\gamma \bar{\psi}^{2} * \kappa_{\delta}(y)\right) \mathrm{d} y \rightarrow \int H\left(\gamma \bar{\psi}^{2}(y)\right) \mathrm{d} y$ as $\delta \downarrow 0$, uniformly in $\gamma$ on any compact subset of $[0, \infty)$. As a consequence, we have $\lim _{\delta \downarrow 0} \Phi_{H}\left(\bar{\psi}^{2} * \kappa_{\delta}, u\right)=\Phi_{H}\left(\bar{\psi}^{2}, u\right)$ and therefore

$$
\begin{aligned}
\limsup _{\delta \downarrow 0} K_{H}(u ; \delta) & \leqslant \frac{1}{2}\left\|\Gamma^{1 / 2} \nabla \bar{\psi}\right\|_{2}^{2}+\limsup _{\delta \downarrow 0} \Phi_{H}\left(\bar{\psi}^{2} * \kappa_{\delta}, u\right)=\frac{1}{2}\left\|\Gamma^{1 / 2} \nabla \bar{\psi}\right\|_{2}^{2}+\Phi_{H}\left(\bar{\psi}^{2}, u\right) \\
& \leqslant K_{H}(u)+\varepsilon .
\end{aligned}
$$

Now let $\varepsilon \downarrow 0$.

Lemma 3.6 implies the corresponding statement for the case $(\mathrm{V})$ :

Corollary 3.7 (Approximations of $K_{D, q}$ ). Fix $D>0$ and $q>1$ and recall that $\frac{1}{p}+\frac{1}{q}=1$. Then

$$
\limsup _{R \rightarrow \infty} K_{D, q}^{(0)}(R) \leqslant K_{D, q} \leqslant \liminf _{\delta \downarrow 0} \liminf _{R \rightarrow \infty} K_{D, q}^{(\mathrm{per})}(\delta, R),
$$

where

$$
\begin{aligned}
& K_{D, q}^{(0)}(R)=\inf \left\{\frac{1}{2}\left\|\Gamma^{1 / 2} \nabla \psi\right\|_{2}^{2}+D\left\|\psi^{2}\right\|_{p}^{-q}: \psi \in H^{1}\left(\mathbb{R}^{d}\right), \operatorname{supp}(\psi) \subset Q_{R},\|\psi\|_{2}=1\right\}, \\
& K_{D, q}^{\text {(per) }}(\delta, R)=\inf \left\{\frac{1}{2}\left\|\Gamma^{1 / 2} \nabla_{R} \psi\right\|_{2}^{2}+D\left\|\psi^{2} * \kappa_{\delta}\right\|_{p}^{-q}: \psi \in H^{1}\left(Q_{R}\right),\|\psi\|_{2}=1\right\},
\end{aligned}
$$

and $\nabla_{R}$ is the gradient on the torus $Q_{R}$, i.e., with periodic boundary condition. 
Proof. We apply Lemma 3.6 to the special choice $u=1$ and $H(t)=\widetilde{D} t^{p}$, where $p$ and $\widetilde{D}$ are as in (1.8). It is easy to see that for this choice of $H$, we have $\Phi_{H}\left(\psi^{2}, 1\right)=D\left\|\psi^{2}\right\|_{p}^{-q}$.

\section{Proof of the upper bounds in Theorems 1.1 and 1.3}

This section is devoted to the proof of the upper bounds in Theorems 1.1 and 1.3. They are in Sections 4.1 and 4.2, respectively. Our proofs essentially follow the proof of [1, Theorem 2.2].

\subsection{Very-large deviation case (Theorem 1.1)}

In this section we are under Assumption (Y) with $q>\frac{d}{2}$, and consider a sequence $\left(b_{n}\right)$ with $1 \ll b_{n} \ll n^{1 / q}$. We have to smoothen the scenery, as we have explained at the beginning of Section 3. In order to do this, we have to cut down the scenery to bounded size. As soon as the smoothing argument has been carried out, we may relax the boundedness assumption.

Recall the scaled and normalized local times $L_{n}$ from (1.19) and the scaled normalized scenery $\bar{Y}_{n}$ from (1.21). Recall the notation $y^{(\leqslant M)}=[y \wedge M] \vee(-M)$ from (3.10), and recall the delta-approximation $\kappa_{\delta}: \mathbb{R}^{d} \rightarrow[0, \infty)$ to the Dirac measure from the beginning of Section 3.4.

Note that, for any $M, \varepsilon, \delta>0$,

$$
\begin{aligned}
\mathbb{P}\left(\frac{1}{n} Z_{n}>b_{n}\right) \leqslant & \mathbb{P}\left(\left\langle\bar{Y}_{n}^{(\leqslant M)} * \kappa_{\delta}, L_{n}\right\rangle>1-2 \varepsilon\right)+\mathbb{P}\left(\left\langle\left|\bar{Y}_{n}^{(\leqslant M)} * \kappa_{\delta}-\bar{Y}_{n}^{(\leqslant M)}\right|, L_{n}\right\rangle>\varepsilon\right) \\
& +\mathbb{P}\left(\left\langle\bar{Y}_{n}^{(>M)}, L_{n}\right\rangle>\varepsilon\right) .
\end{aligned}
$$

Recall that, by our choice of $\alpha_{n}$, we have

$$
n^{d /(d+2)} b_{n}^{2 q /(d+2)}=\frac{n}{\alpha_{n}^{2}} .
$$

Hence, by Proposition 3.4, Lemma 3.5 and Corollary 3.7, it suffices to prove, for any $M, \delta>0$ and $R \in \mathbb{N}$,

$$
\underset{\varepsilon \downarrow 0}{\limsup } \limsup _{n \rightarrow \infty} \frac{\alpha_{n}^{2}}{n} \log \mathbb{P}\left(\left\langle\bar{Y}_{n}^{(\leqslant M)} * \kappa_{\delta}, L_{n}\right\rangle>1-2 \varepsilon\right) \leqslant-K_{D, q}^{\text {(per) }}(\delta, R),
$$

where $K_{D, q}^{(\mathrm{per})}(\delta, R)$ is defined in Corollary 3.7. Note that

$$
\begin{aligned}
\left\langle\bar{Y}_{n}^{(\leqslant M)} * \kappa_{\delta}, L_{n}\right\rangle & =\frac{1}{b_{n} \alpha_{n}^{d}} \sum_{z \in \mathbb{Z}^{d}}\left[\left(Y(z) \wedge\left(M b_{n}\right)\right) \vee\left(-M b_{n}\right)\right] L_{n} * \kappa_{\delta}\left(\frac{z}{\alpha_{n}}\right) \\
& \leqslant \frac{1}{b_{n} \alpha_{n}^{d}} \sum_{z \in \mathbb{Z}^{d}}\left[Y(z) \vee\left(-M b_{n}\right)\right] L_{n} * \kappa_{\delta}\left(\frac{z}{\alpha_{n}}\right) .
\end{aligned}
$$

Introduce the cumulant generating function of $Y(0) \vee(-M)$,

$$
H_{M}(t)=\log \mathbb{E}\left[\mathrm{e}^{t[Y(0) \vee(-M)]}\right] .
$$

Using the exponential Chebyshev inequality and carrying out the expectation over the scenery, we obtain, for any $\gamma>0$, the upper bound

$$
\begin{aligned}
\mathbb{P}\left(\left\langle\bar{Y}_{n}^{(\leqslant M)} * \kappa_{\delta}, L_{n}\right\rangle>1-2 \varepsilon\right) & \leqslant \mathbb{E}\left[\mathrm{e}^{-\gamma(1-2 \varepsilon) n b_{n}} \mathrm{e}^{\gamma n \alpha_{n}^{-d} \sum_{z}\left[Y(z) \vee\left(-M b_{n}\right)\right] L_{n} * \kappa_{\delta}\left(z / \alpha_{n}\right)}\right] \\
& \leqslant \mathbb{E}\left[\mathrm{e}^{-\gamma(1-2 \varepsilon) n b_{n}} \exp \left\{\sum_{z \in \mathbb{Z}^{d}} H_{M b_{n}}\left(\gamma n \alpha_{n}^{-d} L_{n} * \kappa_{\delta}\left(\frac{z}{\alpha_{n}}\right)\right)\right\}\right] .
\end{aligned}
$$

Since $H_{M b_{n}}$ is convex and satisfies $H_{M b_{n}}(0)=0$, it is also superadditive. Hence, for any $\gamma>0$ and any $x \in \mathbb{Z}^{d}$, we have

$$
\sum_{k \in \mathbb{Z}^{d}} H_{M b_{n}}\left(\gamma \ell_{n}(x+2 k\lfloor R\rfloor)\right) \leqslant H_{M b_{n}}\left(\gamma \ell_{n}^{(R)}(x)\right) .
$$


Therefore, the right-hand side in (4.4) does not become smaller if $L_{n} * \kappa_{\delta}$ is replaced by its periodized version, $\left(L_{n} * \kappa_{\delta}\right)^{(R)}(x)=\sum_{k \in \mathbb{Z}^{d}} L_{n} * \kappa_{\delta}(x+k R)$, for $x \in[-R, R]^{d}$. Furthermore, note that

$$
\left(L_{n} * \kappa_{\delta}\right)^{(R)}(x)=\sum_{k \in \mathbb{Z}^{d}} \int_{\mathbb{R}^{d}} L_{n}(y) \kappa_{\delta}(x+k R-y) \mathrm{d} y=\int_{\mathbb{R}^{d}} L_{n}^{(R)}(y) \kappa_{\delta}(x-y) \mathrm{d} y=L_{n}^{(R)} * \kappa_{\delta}(x),
$$

for any $x \in[-R, R]^{d}$. Hence, we may replace $L_{n}$ on the right of (4.4) by its periodized version $L_{n}^{(R)}$.

According to (1.8), for any $\varepsilon>0$, we may choose a $c(\varepsilon)>0$ such that

$$
H(t) \leqslant c(\varepsilon) t+(1+\varepsilon) \widetilde{D} t^{p}, \quad t \in[0, \infty) .
$$

Since $\mathrm{e}^{H_{M}(t)} \leqslant \mathrm{e}^{H(t)}+1$, we also have the estimate in (4.6) for $H_{M b_{n}}$ instead of $H$. Hence, since $\kappa_{\delta}$ and $L_{n}$ are $L^{1}$-normalized,

$$
\mathbb{P}\left(\left\langle\bar{Y}_{n}^{(\leqslant M)} * \kappa_{\delta}, L_{n}\right\rangle>1-2 \varepsilon\right) \leqslant \mathbb{E}\left[\mathrm{e}^{-\gamma(1-2 \varepsilon) n b_{n}} \mathrm{e}^{c(\varepsilon) \gamma n} \exp \left\{\gamma^{p}(\widetilde{D}+\varepsilon) \alpha_{n}^{d}\left(n \alpha_{n}^{-d}\right)^{p}\left\|L_{n}^{(R)} * \kappa_{\delta}\right\|_{p}^{p}\right\}\right] .
$$

We choose the value of $\gamma$ optimal for $\varepsilon=0$, which is

$$
\gamma=\frac{\alpha_{n}^{d}}{n} b_{n}^{1 /(p-1)}\left(p \widetilde{D}\left\|L_{n} * \kappa_{\delta}^{p}\right\|_{p}^{p}\right)^{-1 /(p-1)}=\frac{\alpha_{n}^{d}}{n} b_{n}^{1 /(p-1)} D q\left\|L_{n}^{(R)} * \kappa_{\delta}\right\|_{p}^{-q},
$$

where we recalled that $1=\frac{1}{p}+\frac{1}{q}$ and $\widetilde{D}=(q-1)\left(D q^{q}\right)^{1 /(1-q)}$. Note that the map $\mu \mapsto\left\|\mu * \kappa_{\delta}\right\|_{p}^{-q}$ is bounded and continuous (in the weak $L^{1}$-topology) on the set of probability measures on $[-R, R]^{d}$. Indeed, the continuity is seen with the help of Lebesgue's theorem, and the boundedness follows from the following application of Jensen's inequality:

$$
\begin{aligned}
\left\|\mu * \kappa_{\delta}\right\|_{p}^{p} & =(2 R)^{d} \int_{[-R, R]^{d}} \frac{\mathrm{d} x}{(2 R)^{d}}\left|\int_{\mathbb{R}^{d}} \mu(\mathrm{d} y) \kappa_{\delta}(x-y)\right|^{p} \\
& \geqslant(2 R)^{d}\left(\int_{[-R, R]^{d}} \frac{\mathrm{d} x}{(2 R)^{d}} \int_{\mathbb{R}^{d}} \mu(\mathrm{d} y) \kappa_{\delta}(x-y)\right)^{p} \\
& =(2 R)^{d(1-p)}
\end{aligned}
$$

since $\kappa_{\delta}$ is $L^{1}$-normalized.

Recall that $b_{n}^{q}=n \alpha_{n}^{-(d+2)}$. For the choice of $\gamma$ in (4.8), for large $n$, we can estimate the first two terms in the expectation on the right of (4.7) by $\mathrm{e}^{-\gamma(1-2 \varepsilon) n b_{n}} \mathrm{e}^{c(\varepsilon) \gamma n} \leqslant \mathrm{e}^{-\gamma(1-3 \varepsilon) n b_{n}}$, since we have in particular $\gamma \ll b_{n}$.

Substituting $\gamma$ in (4.7), we obtain

$$
\mathbb{P}\left(\left\langle\bar{Y}_{n}^{(\leqslant M)} * \kappa_{\delta}, L_{n}\right\rangle>1-2 \varepsilon\right) \leqslant \mathbb{E}\left[\exp \left\{-(D+\varepsilon C) \frac{n}{\alpha_{n}^{2}}\left\|L_{n}^{(R)} * \kappa_{\delta}\right\|_{p}^{-q}\right\}\right]
$$

where $C>0$ depends on $D, R$ and $q$ only. Now we can finally apply the large deviation principle in Lemma 3.1(ii) to the right-hand side of (4.10). This yields the estimate in (4.3) without $\lim \sup _{\varepsilon \downarrow 0}$ and with $D$ replaced by $D+\varepsilon C$. Letting $\varepsilon \downarrow 0$, we easily see that (4.3) is satisfied, which ends the proof of the upper bound in Theorem 1.1.

\subsection{Large-deviation case (Theorem 1.3)}

In this section, we prove the upper bound in Theorem 1.3, i.e., in the case (L). The proof follows the pattern of the corresponding proof in [1] and is analogous to the proof of Theorem 1.1 in Section 4.1, and hence we keep it short. Pick $b_{n}=1$ and $\alpha_{n}=n^{1 /(d+2)}$, in accordance with (1.27). Furthermore, fix $u>0$.

By Proposition 3.4 and Lemmas 3.5 and 3.6, it is sufficient to prove that, for any $\delta>0$ and $R \in \mathbb{N}$,

$$
\underset{\varepsilon \downarrow 0}{\limsup } \limsup _{n \rightarrow \infty} n^{-d /(d+2)} \log \mathbb{P}\left(\left\langle\bar{Y}_{n}^{(\leqslant M)} * \kappa_{\delta}, L_{n}\right\rangle>u-\varepsilon\right) \leqslant-K_{H}^{(\mathrm{per})}(u ; \delta, R),
$$


where $K_{H}^{(\mathrm{per})}(u ; \delta, R)$ is defined in Lemma 3.6. Fix a small $\varepsilon>0$. Analogously to (4.4), we have the estimate

$$
\mathbb{P}\left(\left\langle\bar{Y}_{n}^{(\leqslant M)} * \kappa_{\delta}, L_{n}\right\rangle>u-\varepsilon\right) \leqslant \mathbb{E}\left[\mathrm{e}^{-\gamma(u-2 \varepsilon) n} \exp \left\{\sum_{z \in \mathbb{Z}^{d}} H_{M}\left(\gamma n \alpha_{n}^{-d} L_{n}^{(R)} * \kappa_{\delta}\left(\frac{z}{\alpha_{n}}\right)\right)\right\}\right]
$$

for any $\gamma>0$. Replacing $\gamma n \alpha_{n}^{-d}$ by $\gamma$, turning the sum into an integral, passing to the optimum over $\gamma$ and using the notation in (3.9), we obtain

$$
\mathbb{P}\left(\left\langle\bar{Y}_{n}^{(\leqslant M)} * \kappa_{\delta}, L_{n}\right\rangle>u\right) \leqslant \mathbb{E}\left[\exp \left\{-\frac{n}{\alpha_{n}^{2}} \Phi_{H_{M}}\left(L_{n}^{(R)} * \kappa_{\delta}, u-2 \varepsilon ; R\right)\right\}\right],
$$

where we also recall that $\alpha_{n}^{d}=n \alpha_{n}^{-2}$. Again, for fixed $\delta>0$ and $R>0$, we can let $M \rightarrow \infty$ and $\varepsilon \downarrow 0$ to replace $\Phi_{H_{M}}\left(L_{n}^{(R)} * \kappa_{\delta}, u-2 \varepsilon ; R\right)$ by $\Phi_{H}\left(L_{n}^{(R)} * \kappa_{\delta}, u ; R\right)$ on the right side of (4.13). Analogously to (4.9), one shows that $\Phi_{H}\left(\psi^{2}, u\right) \leqslant\left|Q_{R}\right| \sup _{\gamma>0}(\gamma u-H(\gamma))<\infty$ for any continuous $\psi: Q_{R} \rightarrow[0, \infty)$ satisfying $\int_{Q_{R}} \psi^{2}=1$. Hence, the map $\mu \mapsto \Phi_{H}\left(\mu * \kappa_{\delta}, u ; R\right)$ is bounded and continuous on the set of probability measures on $Q_{R}$, and we may apply the large deviation principle in Lemma 3.1(ii). This, followed by $\varepsilon \downarrow 0$, implies that (4.11) holds for any $\delta>0$ and $R \in \mathbb{N}$. This finishes the proof of the upper bound in Theorem 1.3.

\section{Proofs of the lower bounds in Theorems 1.1 and 1.3}

In this section we prove the lower bounds in Theorems 1.1 and 1.3. Our proofs are variants of the analogous proofs in [1]; they roughly follow the heuristics in Section 1.3.

\subsection{Very-large deviation case (Theorem 1.1)}

Suppose we are in the case (V) and pick sequences $\left(b_{n}\right)_{n}$ and $\left(\alpha_{n}\right)_{n}$ as in (1.27). Fix $R>0$ and any continuous positive function $\varphi: Q_{R} \rightarrow(0, \infty)$. Recall the scaled local times and scenery, $L_{n}$ and $\bar{Y}_{n}$, in (1.19) and (1.21).

If $\bar{Y}_{n} \geqslant \varphi$ on $Q_{R}$ and $\operatorname{supp}\left(L_{n}\right) \subset Q_{R}$, then

$$
Z_{n}=b_{n} n\left\langle L_{n}, \bar{Y}_{n}\right\rangle \geqslant b_{n} n\left\langle L_{n}, \varphi\right\rangle \text {. }
$$

Hence, we obtain the lower bound, for any $n \in \mathbb{N}$,

$$
\mathbb{P}\left(\frac{1}{n} Z_{n}>b_{n}\right) \geqslant \mathbb{P}\left(\left\langle L_{n}, \varphi\right\rangle \geqslant 1, \operatorname{supp}\left(L_{n}\right) \subset Q_{R}\right) \mathbb{P}\left(\bar{Y}_{n} \geqslant \varphi \text { on } Q_{R}\right) .
$$

With the help of the large deviation principle in Lemma 3.1(i) it is easy to deduce that

$$
\begin{aligned}
& \lim _{n \rightarrow \infty} \frac{\alpha_{n}^{2}}{n} \log \mathbb{P}\left(\left\langle L_{n}, \varphi\right\rangle \geqslant 1, \operatorname{supp}\left(L_{n}\right) \subset Q_{R}\right) \\
& \quad=-\inf \left\{\mathcal{I}_{R}\left(\psi^{2}\right): \psi \in H^{1}\left(\mathbb{R}^{d}\right), \operatorname{supp}(\psi) \subset Q_{R},\|\psi\|_{2}=1,\left\langle\psi^{2}, \varphi\right\rangle \geqslant 1\right\} .
\end{aligned}
$$

From Lemma 3.2 we have, recalling that $n \alpha_{n}^{-2}=\alpha_{n}^{d} b_{n}^{q}$,

$$
\liminf _{n \rightarrow \infty} \frac{\alpha_{n}^{2}}{n} \log \mathbb{P}\left(\bar{Y}_{n} \geqslant \varphi \text { on } Q_{R}\right) \geqslant-D\|\varphi\|_{q}^{q} \text {. }
$$

Using (5.3) and (5.4) in (5.2) and optimizing on $\varphi$, we obtain the lower bound

$$
\liminf _{n \rightarrow \infty} \frac{\alpha_{n}^{2}}{n} \log \mathbb{P}\left(\frac{1}{n} Z_{n}>b_{n}\right) \geqslant-\widetilde{K}_{D, q}^{(0)}(R),
$$

where

$$
\tilde{K}_{D, q}(R)=\inf _{\psi \in H^{1}\left(\mathbb{R}^{d}\right):\|\psi\|_{2}=1, \operatorname{supp}(\psi) \subset B_{R}}\left(\mathcal{I}_{R}\left(\psi^{2}\right)+D \inf _{\varphi \in \mathcal{C}_{+}\left(Q_{R}\right):\left\langle\psi^{2}, \varphi\right\rangle \geqslant 1}\|\varphi\|_{q}^{q}\right) .
$$

It is easy to see that the inner infimum is equal to $\left\|\psi^{2}\right\|_{p}^{-q}$. Hence, $\widetilde{K}_{D, p}(R)=K_{D, p}^{(0)}(R)$ as defined in Corollary 3.7. Now Corollary 3.7 finishes the proof of the lower bound in Theorem 1.1. 


\subsection{Large-deviation case (Theorem 1.3)}

Recall from Section 1.3 that $\frac{1}{n} Z_{n}=\left\langle L_{n}, \bar{Y}_{n}\right\rangle$. We want to apply the large deviation principles of Lemma 3.1(i) for $L_{n}$ and Lemma 3.3 for $\bar{Y}_{n}$. However, as has been pointed out in [1], the map $(\mu, f) \mapsto\langle\mu, f\rangle$ is not continuous in the product of the weak topologies. Hence, we partially follow the strategy of [1] and use Lemma 3.5 to smoothen the field $\bar{Y}_{n}$. In order to apply Lemma 3.5, we first have to cut down the field to bounded size, which we do with the help of Proposition 3.4. However, this works only for cutting the large values of the field, but not the small ones. In order to be able to use also a lower bound for the field, we intersect with the event that $Y(z) \geqslant-M$ for all $z$ 's appearing, and use a large deviation principle for the conditional field.

Let us turn to the details. Let $u>0$ satisfying $u \in \operatorname{supp}(Y(0))^{\circ}$. We fix small parameter $\varepsilon, \delta>0$ such that $u+\varepsilon \in$ $\operatorname{supp}(Y(0))^{\circ}$ and large parameters $M$ and $R$. On the intersection of the events $\left\{\operatorname{supp}\left(L_{n}\right) \subset Q_{R}\right\}$ and $\{Y(z) \geqslant-M$ $\left.\forall z \in B_{R \alpha_{n}}\right\}$, we can estimate

$$
\frac{1}{n} Z_{n}=\left\langle L_{n}, \bar{Y}_{n}\right\rangle \geqslant\left\langle L_{n}, \bar{Y}_{n}^{(\leqslant M)}\right\rangle=\left\langle L_{n} * \kappa_{\delta}, \bar{Y}_{n}^{(\leqslant M)}\right\rangle+\left\langle L_{n}, \bar{Y}_{n}^{(\leqslant M)}-\bar{Y}_{n}^{(\leqslant M)} * \kappa_{\delta}\right\rangle .
$$

We write $\mathbb{P}^{(>-M)}$ for the conditional measure $\mathbb{P}\left(\cdot \mid Y(z) \geqslant-M \forall z \in \mathbb{Z}^{d}\right)$. Hence, we obtain the lower bound

$$
\begin{aligned}
\mathbb{P}\left(\frac{1}{n} Z_{n}>u\right) \geqslant & \mathbb{P}^{(>-M)}\left(\operatorname{supp}\left(L_{n}\right) \subset Q_{R},\left\langle L_{n} * \kappa_{\delta}, \bar{Y}_{n}^{(\leqslant M)}\right\rangle>u+\varepsilon\right) \mathbb{P}(Y(0) \geqslant-M)^{\left|B_{R \alpha_{n}}\right|} \\
& -\mathbb{P}\left(\left\langle L_{n}, \bar{Y}_{n}^{(\leqslant M)}-\bar{Y}_{n}^{(\leqslant M)} * \kappa_{\delta}\right\rangle>\varepsilon\right) .
\end{aligned}
$$

Using Lemma 3.5 for the last term on the right-hand side, and noting that $\mathbb{P}(Y(0) \geqslant-M) \rightarrow 0$ as $M \rightarrow \infty$, it becomes clear that it suffices to estimate the first term on the right side. In order to do this, fix a positive continuous function $g: Q_{R} \rightarrow(0, \infty)$ satisfying $\int_{Q_{R}} g(x) \mathrm{d} x=1$ such that $g$ can be extended to an element of $H^{1}\left(\mathbb{R}^{d}\right)$. Let $B_{\varepsilon}(g)$ denote a weak $\varepsilon$-neighborhood of $g$. Then we have

$$
\begin{aligned}
& \mathbb{P}^{(>-M)}\left(\operatorname{supp}\left(L_{n}\right) \subset Q_{R},\left\langle L_{n} * \kappa_{\delta}, \bar{Y}_{n}^{(\leqslant M)}\right\rangle>u+\varepsilon\right) \\
& \quad \geqslant \mathbb{P}\left(L_{n} \in B_{\varepsilon}(g), \operatorname{supp}\left(L_{n}\right) \subset Q_{R}\right) \mathbb{P}^{(>-M)}\left(\left\langle g * \kappa_{\delta}, \bar{Y}_{n}^{(\leqslant M)}\right\rangle>u+2 \varepsilon\right) .
\end{aligned}
$$

According to Lemma 3.1, the first term on the right is equal to $\exp \left\{-n \alpha_{n}^{-2} \inf _{\psi^{2} \in B_{\varepsilon}(g)} \mathcal{I}_{R}\left(\psi^{2}\right)(1+\mathrm{o}(1))\right\}$, and according to Lemma 3.3, the latter term is equal to $\exp \left\{-n \alpha_{n}^{-2} \Phi_{\widetilde{H}_{M}}\left(g * \kappa_{\delta}, u-2 \varepsilon, R\right)(1+\mathrm{o}(1))\right\}$. Summarizing, we obtain, for any $R>0$ and any continuous positive function $g: Q_{R} \rightarrow(0, \infty)$, if $M$ is sufficiently large and $\delta>0$ sufficiently small,

$$
\liminf _{n \rightarrow \infty} \frac{\alpha_{n}^{2}}{n} \log \mathbb{P}\left(\frac{1}{n} Z_{n}>u\right) \geqslant-\left[\mathcal{I}_{R}(g)+\Phi_{\widetilde{H}_{M}}\left(g * \kappa_{\delta}, u+2 \varepsilon, R\right)\right]+\eta_{M},
$$

for some $\eta_{M} \downarrow 0$ as $M \rightarrow \infty$. Passing to the infimum over all $g$ and writing $\psi^{2}$ instead of $g$, we obtain

$$
\liminf _{n \rightarrow \infty} \frac{\alpha_{n}^{2}}{n} \log \mathbb{P}\left(\frac{1}{n} Z_{n}>u\right) \geqslant-\inf _{\psi \in H^{1}\left(\mathbb{R}^{d}\right): \operatorname{supp}(\psi) \subset Q_{R}}\left[\mathcal{I}_{R}\left(\psi^{2}\right)+\Phi_{\widetilde{H}_{M}}\left(\psi^{2} * \kappa_{\delta}, u+2 \varepsilon, R\right)\right]+\eta_{M} .
$$

Since $\psi^{2} * \kappa_{\delta}$ is bounded uniformly in $\psi$, and since $\widetilde{H}_{M}(t) \rightarrow H(t)$ as $M \rightarrow \infty$, uniformly in $t$ on compacts, we can let $M \rightarrow \infty$. Furthermore, we also let $\varepsilon \downarrow 0$ and obtain

$$
\liminf _{n \rightarrow \infty} \frac{\alpha_{n}^{2}}{n} \log \mathbb{P}\left(\frac{1}{n} Z_{n}>u\right) \geqslant-K_{H}(u ; \delta, R),
$$

for any $\delta>0$ and $R>0$, where $K_{H}^{(0)}(u ; \delta, R)$ is defined in Lemma 3.6. Now use Lemma 3.6 to finish the proof of the lower bound in Theorem 1.3.

\section{Acknowledgements}

This work was partially supported by DFG grant Ko 2205/1-1. W. K. thanks the Deutsche Forschungsgemeinschaft for awarding a Heisenberg grant (realized in 2003/04). W. K. and N. G. thank the Laboratoire de Probabilités for its hospitality. All three authors thank Francis Comets for helpful discussions. We also thank the referee for carefully reading the first version of the paper. 


\section{Appendix A. Proof of the large deviation principle for the local times}

In this section, we prove the scaled large deviation principles in Lemma 3.1. Although the statement should be familiar to experts and the proof is fairly standard, we could not find it in the literature. Therefore, we provide a proof. Let us mention that the lower bound of the following Lemma A.1 (without the indicator on $\left\{\operatorname{supp}\left(L_{n}\right) \subset Q_{R}\right\}$, however) is contained in [13].

Fix $R>0$. For bounded and continuous functions $f: Q_{R} \rightarrow \mathbb{R}$, we denote by

$$
\lambda_{R}(f)=\max \left\{\left\langle f, \psi^{2}\right\rangle-\frac{1}{2}\left\|\Gamma^{1 / 2} \nabla \psi\right\|_{2}^{2}: \psi \in H^{1}\left(\mathbb{R}^{d}\right), \operatorname{supp}(\psi) \subset Q_{R},\|\psi\|_{2}=1\right\}
$$

the principal eigenvalue of the operator $\frac{1}{2} \nabla \cdot \Gamma \nabla+f$ in $Q_{R}$ with Dirichlet boundary condition. (We denote the inner product and norm on $L^{2}\left(Q_{R}\right)$ by $\langle\cdot, \cdot\rangle$ and $\|\cdot\|_{2}$.) The main step in the proof of Lemma 3.1(i) is the following.

Lemma A.1. For any bounded and continuous function $f: Q_{R} \rightarrow \mathbb{R}$, the limit

$$
\lim _{n \rightarrow \infty} \frac{\alpha_{n}^{2}}{n} \log \mathbb{E}\left[\exp \left\{\frac{n}{\alpha_{n}^{2}}\left\langle f, L_{n}\right\rangle\right\} \mathbb{1}\left\{\operatorname{supp}\left(L_{n}\right) \subset Q_{R}\right\}\right]
$$

exists and is equal to $\lambda_{R}(f)$.

Proof. In the following, we abbreviate $B=B_{R \alpha_{n}}$. Introduce a scaled version $f_{n}: \mathbb{Z}^{d} \rightarrow \mathbb{R}$ of $f$ by

$$
f_{n}(z)=\alpha_{n}^{d} \int_{z \alpha_{n}^{-1}+\left[0, \alpha_{n}^{-1}\right)^{d}} f(x) \mathrm{d} x, \quad z \in \mathbb{Z}^{d} .
$$

Note that $f_{n}\left(\left\lfloor\cdot \alpha_{n}\right\rfloor\right) \rightarrow f$ uniformly on $Q_{R}$. Furthermore, note that

$$
\frac{n}{\alpha_{n}^{2}}\left\langle f, L_{n}\right\rangle=\alpha_{n}^{d-2} \int_{Q_{R}} f(x) \ell_{n}\left(\left\lfloor x \alpha_{n}\right\rfloor\right) \mathrm{d} x=\alpha_{n}^{-2} \sum_{z \in B} \ell_{n}(z) f_{n}(z)=\sum_{k=0}^{n-1} \alpha_{n}^{-2} f_{n}\left(S_{k}\right) .
$$

For notational convenience, we assume that $\alpha_{n}^{2}$ and $n \alpha_{n}^{-2}$ are integers. Using the Markov property, we split the expectation over the path $\left(S_{0}, \ldots, S_{n}\right)$ into $n \alpha_{n}^{-2}$ expectations over paths of length $\alpha_{n}^{2}$. By $\mathbb{E}_{z}$ we denote the expectation with respect to the random walk starting at $z \in \mathbb{Z}^{d}$, then we have

$$
\begin{aligned}
\mathbb{E} & {\left[\exp \left\{\frac{n}{\alpha_{n}^{2}}\left\langle f, L_{n}\right\rangle\right\} \mathbb{1}\left\{\operatorname{supp}\left(L_{n}\right) \subset Q_{R}\right\}\right] } \\
= & \mathbb{E}\left[\exp \left\{\frac{1}{\alpha_{n}^{2}} \sum_{k=0}^{n-1} f_{n}\left(S_{k}\right)\right\} \mathbb{1}\left\{\operatorname{supp}\left(\ell_{n}\right) \subset B\right\}\right] \\
= & \sum_{z_{1}, \ldots, z_{n \alpha_{n}^{-2}} \in B} \prod_{i=1}^{n \alpha_{n}^{-2}} \mathbb{E}_{z_{i-1}}\left[\exp \left\{\frac{1}{\alpha_{n}^{2}} \sum_{k=0}^{\alpha_{n}^{2}-1} f_{n}\left(S_{k}\right)\right\} \mathbb{1}\left\{\operatorname{supp}\left(\ell_{\alpha_{n}^{2}}\right) \subset B\right\} \mathbb{1}\left\{S_{\alpha_{n}^{2}}=z_{i}\right\}\right] \\
= & \int \mathrm{d} x_{1} \cdots \mathrm{d} x_{n \alpha_{n}^{-2}} \prod_{i=1}^{n \alpha_{n}^{-2}}\left[\alpha_{n}^{d} \mathbb{E}_{\left\lfloor x_{i-1} \alpha_{n}\right\rfloor}\left[\exp \left\{\frac{1}{\alpha_{n}^{2}} \sum_{k=0}^{\alpha_{n}^{2}-1} f_{n}\left(S_{k}\right)\right\} \mathbb{1}\left\{\operatorname{supp}\left(\ell_{\alpha_{n}^{2}}\right) \subset B\right\} \mathbb{1}\left\{S_{\alpha_{n}^{2}}=\left\lfloor x_{i} \alpha_{n}\right\rfloor\right\}\right] .\right.
\end{aligned}
$$

Let $\left(B_{t}\right)_{t} \geqslant 0$ be the Brownian motion on $\mathbb{R}^{d}$ with covariance matrix $\Gamma$, and let $\mathrm{E}_{x}$ denote the corresponding expectation, when $B_{0}=x \in \mathbb{R}^{d}$. Then $\left(\alpha_{n}^{-1} S_{\left\lfloor t \alpha_{n}^{2}\right.}\right)_{t \geqslant 0}$ converges weakly towards $\left(B_{t}\right)_{t \geqslant 0}$ in distribution, and from a local central limit theorem (see [24, P7.9, P7.10]) it follows that, uniformly in $x, y \in Q_{R}$, 


$$
\begin{aligned}
& \lim _{n \rightarrow \infty} \alpha_{n}^{d} \mathbb{E}_{\left\lfloor x \alpha_{n}\right\rfloor}\left[\exp \left\{\frac{1}{\alpha_{n}^{2}} \sum_{k=0}^{\alpha_{n}^{2}-1} f_{n}\left(S_{k}\right)\right\} \mathbb{1}\left\{\operatorname{supp}\left(\ell_{\alpha_{n}^{2}}\right) \subset B\right\} \mathbb{1}\left\{S_{\alpha_{n}^{2}}=\left\lfloor y \alpha_{n}\right\rfloor\right\}\right] \\
& =\mathrm{E}_{x}\left(\exp \left\{\int_{0}^{1} f\left(B_{S}\right) \mathrm{d} s\right\} \mathbb{1}\left\{B_{[0,1]} \subset Q_{R}\right\} ; B_{1} \in \mathrm{d} y\right) / \mathrm{d} y .
\end{aligned}
$$

Substituting this on the right-hand side of (A.5) and again using the Markov property, we obtain that, as $n \rightarrow \infty$,

$$
\mathbb{E}\left[\exp \left\{\frac{n}{\alpha_{n}^{2}}\left\langle f, L_{n}\right\rangle\right\} \mathbb{1}\left\{\operatorname{supp}\left(L_{n}\right) \subset Q_{R}\right\}\right]=\mathrm{e}^{\mathrm{o}\left(n \alpha_{n}^{-2}\right)} \mathrm{E}_{0}\left(\exp \left\{\int_{0}^{n \alpha_{n}^{-2}} f\left(B_{S}\right) \mathrm{d} s\right\} \mathbb{1}\left\{B_{\left[0, n \alpha_{n}^{-2}\right]} \subset Q_{R}\right\}\right) .
$$

It is well known that the expectation on the right-hand side of (A.7) is equal to $\exp \left\{\frac{n}{\alpha_{n}^{2}}\left[\lambda_{R}(f)+\mathrm{o}(1)\right]\right\}$ as $n \rightarrow \infty$, and this ends the proof of Lemma A.1.

Proof of Lemma 3.1(i). We shall apply a version of the abstract Gärtner-Ellis theorem (see [14, Section 4.5]). (There is no problem in applying that result for subprobability measures instead of probability measure.) More precisely, we shall apply [14, Corollary 4.5.27], which implies the statement of Lemma 3.1(i) under the following two assumptions: (1) the distributions of $L_{n}$ under $\mathbb{P}\left(\cdot, \operatorname{supp}\left(L_{n}\right) \subset Q_{R}\right)$ form an exponentially tight family, and (2) the limit in (A.2) exists and is a finite, Gâteau-differentiable and lower semicontinuous function of $f$. These two points are satisfied in our case. Indeed, (1) is trivially satisfied since we consider subprobability measures on a compact set $Q_{R}$, and (2) follows from Lemma A.1, together with [17], where the Gâteau-differentiability and lower semicontinuity of the map $f \mapsto \lambda_{R}(f)$ is shown. An application of [14, Corollary 4.5.27] therefore yields the validity of a large deviation principle as stated in Lemma 3.1(i).

It remains to identify the rate function obtained in [14, Corollary 4.5.27] with the rate function of Lemma 3.1(i), $\mathcal{I}_{R}$. The rate function appearing in [14, Corollary 4.5.27], $\widetilde{\mathcal{I}}_{R}$, is the Legendre transform of $\lambda_{R}(\cdot)$ :

$$
\widetilde{\mathcal{I}}_{R}\left(\psi^{2}\right)=\sup _{f \in \mathcal{C}\left(Q_{R}\right)}\left[\left\langle\psi^{2}, f\right\rangle-\lambda_{R}\left(\psi^{2}\right)\right], \quad \psi^{2} \in \mathcal{F}_{R}
$$

It is obvious from (A.1) that $\lambda_{R}(\cdot)$ is itself the Legendre transform of $\mathcal{I}_{R}$, since $\mathcal{I}_{R}$ is equal to $\infty$ outside $\mathcal{F}_{R}$. Because of the convexity inequality for gradients (see [21, Theorem 7.8]), $\mathcal{I}_{R}$ is a convex function on $\mathcal{F}_{R}$. According to the Duality Lemma [14, Lemma 4.5.8], the Legendre transform of $\lambda_{R}(\cdot)$ is equal to $\mathcal{I}_{R}$, i.e., we have that $\widetilde{\mathcal{I}}_{R}=\mathcal{I}_{R}$. This finishes the proof of Lemma 3.1(i).

Proof of Lemma 3.1(ii). This is a modification of the proof of part (i) above; we point out the differences only. Recall that we identify the box $B_{R}=\{\lfloor-R\rfloor+1, \ldots,\lfloor R\rfloor-1\}^{d}$ with the torus $\{\lfloor-R\rfloor+1, \ldots,\lfloor R\rfloor\}^{d}$ where $\lfloor R\rfloor$ is identified with $\lfloor-R\rfloor+1$. Analogously, we conceive $Q_{R}=[-R, R]^{d}$ as the $d$-dimensional torus with the opposite sides identified.

For a continuous bounded function $f: Q_{R} \rightarrow \mathbb{R}$, introduce the principal eigenvalue of the operator $\frac{1}{2} \nabla \cdot \Gamma \nabla+f$ on $L^{2}\left(Q_{R}\right)$ with periodic boundary condition:

$$
\lambda^{(R)}(f)=\max \left\{\int_{Q_{R}} f(x) \psi^{2}(x) \mathrm{d} x-\frac{1}{2} \int_{Q_{R}}\left|\Gamma^{1 / 2} \nabla_{R} \psi(x)\right|^{2} \mathrm{~d} x: \psi \in \mathcal{C}_{1}\left(Q_{R}\right), \int_{Q_{R}} \psi^{2}(x) \mathrm{d} x=1\right\},
$$

where we recall that $\nabla_{R}$ is the gradient of the torus $Q_{R}$.

The main step in the proof of Lemma 3.1(ii) is to show that, for any continuous bounded function $f: Q_{R} \rightarrow \mathbb{R}$,

$$
\lambda^{(R)}(f)=\lim _{n \rightarrow \infty} \frac{\alpha_{n}^{2}}{n} \log \mathbb{E}\left[\exp \left\{\frac{n}{\alpha_{n}^{2}}\left\langle f, L_{n}^{\left(R \alpha_{n}\right)}\right\rangle\right\}\right] .
$$


This is done in the same way as in the proof of Lemma A.1, noting that the process $\left(\alpha_{n}^{-1} S_{t \alpha_{n}^{2}}^{\left(R \alpha_{n}\right)}\right)_{t \geqslant 0}$ converges weakly in distribution towards $\left(B_{t}^{(R)}\right)_{t \geqslant 0}$, the Brownian motion with covariance matrix $\Gamma$, wrapped around the torus $Q_{R}$. Also using a local central limit theorem, we obtain, as $n \rightarrow \infty$,

$$
\mathbb{E}\left[\exp \left\{\frac{n}{\alpha_{n}^{2}}\left\langle f, L_{n}^{\left(R \alpha_{n}\right)}\right\rangle\right\}\right]=\mathrm{e}^{\mathrm{o}\left(n \alpha_{n}^{-2}\right)} \mathrm{E}_{0}\left[\exp \left\{\int_{0}^{n \alpha_{n}^{-2}} f\left(B_{s}^{(R)}\right) \mathrm{d} s\right\}\right] .
$$

It is well known that the expectation on the right side is equal to $\exp \left\{\frac{n}{\alpha_{n}^{2}}\left[\lambda^{(R)}(f)+\mathrm{o}(1)\right]\right\}$ as $n \rightarrow \infty$, and this shows that also (A.10) holds. The remainder of the proof of Lemma 3.1(ii) is the same as the proof of Lemma 3.1(i).

\section{References}

[1] A. Asselah, F. Castell, Large deviations for Brownian motion in a random scenery, Probab. Theory Related Fields 126 (2003) $497-527$.

[2] A. Asselah, F. Castell, A note on random walk in random scenery, preprint, 2005.

[3] A. Asselah, F. Castell, Self-intersection times for random walk, and random walk in random scenery in dimension $d \geqslant 5$, preprint, 2005.

[4] G. Ben Arous, R. Léandre, Décroissance exponentielle du noyau de la chaleur sur la diagonale. II, Probab. Theory Related Fields 90 (3) (1991) 377-402.

[5] N.H. Bingham, C.M. Goldie, J.L. Teugels, Regular Variation, Cambridge University Press, Cambridge, 1987.

[6] E. Bolthausen, A central limit theorem for two-dimensional random walks in random sceneries, Ann. Probab. 17 (1989) $108-115$.

[7] A.N. Borodin, Limit theorems for sums of independent random variables defined on a transient random walk, in: Investigations in the Theory of Probability Distributions, IV, Zap. Nauchn. Sem. Leningrad. Otdel. Mat. Inst. Steklov. (LOMI) 85 (1979) 17-29, $237,244$.

[8] A.N. Borodin, A limit theorem for sums of independent random variables defined on a recurrent random walk, Dokl. Akad. Nauk SSSR 246 (4) (1979) $786-787$.

[9] D.C. Brydges, G. Slade, The diffusive phase of a model of self-interacting walks, Probab. Theory Related Fields 103 (1995) $285-315$.

[10] F. Castell, Moderate deviations for diffusions in a random Gaussian shear flow drift, Ann. Inst. H. Poincaré Probab. Statist. 40 (3) (2004) 337-366.

[11] F. Castell, F. Pradeilles, Annealed large deviations for diffusions in a random shear flow drift, Stochastic Process Appl. 94 (2001) 171-197.

[12] X. Chen, Exponential asymptotics and law of the iterated logarithm for intersection local times of random walks, Ann. Probab. 32 (4) (2004).

[13] X. Chen, W. Li, Large and moderate deviations for intersection local times, Probab. Theory Related Fields 128 (2004) $213-254$.

[14] A. Dembo, O. Zeitouni, Large Deviations Techniques and Applications, second ed., Springer, Berlin, 1998.

[15] M. Donsker, S.R.S. Varadhan, On the number of distinct sites visited by a random walk, Comm. Pure Appl. Math. 32 (1979) $721-747$.

[16] N. Gantert, R. van der Hofstad, W. König, Deviations of a random walk in a random scenery with stretched exponential tails, Stochastic Process. Appl. 116 (3) (2006) 480-492.

[17] J. Gärtner, On large deviations from the invariant measure, Theory Probab. Appl. 22 (1) (1977) 24-39.

[18] J.-P. Kahane, Some Random Series of Functions, second ed., Cambridge University Press, Cambridge, 1985.

[19] H. Kesten, F. Spitzer, A limit theorem related to a new class of self-similar processes, Z. Wahrsch. Verw. Geb. 50 (1979) 5-25.

[20] W. König, P. Mörters, Brownian intersection local times: upper tail asymptotics and thick points, Ann. Probab. 30 (2002) $1605-1656$.

[21] E.H. Lieb, M. Loss, Analysis, second ed., Grad. Stud. Math., vol. 14, Amer. Math. Soc., Providence, RI, 2001.

[22] M. McLeod, J. Serrin, Uniqueness of solutions of semilinear Poisson equations, Proc. Natl. Acad. Sci. USA 78 (11) (1981) $6592-6595$.

[23] V.V. Petrov, Sums of Independent Random Variables, Springer, Berlin, 1975.

[24] F. Spitzer, Principles of Random Walk, second ed., Springer, Berlin, 1976.

[25] K. Uchiyama, Green's functions for random walks on $\mathbb{Z}^{N}$, Proc. London Math. Soc. 77 (3) (1998) 215-240.

[26] M.I. Weinstein, Nonlinear Schrödinger equations and sharp interpolation estimates, Comm. Math. Phys. 87 (1983) $567-576$. 